\title{
The galaxy population of the complex cluster system Abell 3921^
}

\author{
Florian Pranger ${ }^{1}$, Asmus Böhm ${ }^{1}$, Chiara Ferrari ${ }^{2}$, Antonaldo Diaferio ${ }^{3,4}$, Richard Hunstead ${ }^{5}$, Sophie Maurogordato ${ }^{2}$, \\ Christophe Benoist ${ }^{2}$, Jarle Brinchmann ${ }^{6}$, and Sabine Schindler ${ }^{1}$ \\ ${ }^{1}$ Institute for Astro- and Particle Physics, University of Innsbruck, Technikerstr. 25/8, 6020 Innsbruck, Austria \\ e-mail: florian.pranger@uibk.ac.at \\ 2 Laboratoire Lagrange, UMR 7293, Université de Nice Sophia Antipolis, CNRS, Observatoire de la Côte d'Azur, 06300 Nice, France \\ 3 Dipartimento di Fisica, Università degli Studi di Torino, via P. Giuria 1, 10125 Torino, Italy \\ ${ }^{4}$ Istituto Nazionale di Fisica Nucleare (INFN), Sezione di Torino, via P. Giuria 1, 10125 Torino, Italy \\ 5 School of Physics, University of Sydney, NSW 2006, Australia \\ ${ }^{6}$ Leiden Observatory, Leiden University, 2300 RA Leiden, The Netherlands
}

Received 21 May 2013 / Accepted 16 July 2013

\begin{abstract}
Context. We present a spectrophotometric analysis of the galaxy population in the area of the merging cluster Abell 3921 at $z=0.093$. Aims. We investigate the impact of the complex cluster environment on galaxy properties such as morphology or star formation rate. Methods. We combine multi-object spectroscopy from the two-degree field (2dF) spectrograph with optical imaging taken with the ESO Wide Field Imager. We carried out a redshift analysis and determine cluster velocity dispersions using biweight statistics. Applying a Dressler-Shectman test we sought evidence of cluster substructure. Cluster and field galaxies were investigated with respect to [OII] and $\mathrm{H} \alpha$ equivalent width, star formation rate, and morphological descriptors, such as concentration index and Gini coefficient. We studied these cluster galaxy properties as a function of clustercentric distance and investigated the spatial distribution of various galaxy types.

Results. Applying the Dressler-Shectman test, we find a third component (A3921-C) in addition to the two main subclusters (A3921-A and A3921-B) that are already known. The re-determined mass ratio between the main components A and B is $\sim 2: 1$. Similar to previous studies of galaxy clusters, we find that a large fraction of the disk galaxies close to the cluster core show no detectable star formation. These are likely systems that are quenched due to ram pressure stripping. Interestingly, we also find quenched spirals at rather large distances of 3-4 Mpc from the cluster core.

Conclusions. A3921-C might be a group of galaxies falling onto the main cluster components. We speculate that the unexpected population of quenched spirals at large clustercentric radii in A3921-A and A3921-B might be an effect of the ongoing cluster merger: shocks in the ICM might give rise to enhanced ram pressure stripping and at least in part be the cause for the quenching of star formation. These quenched spirals might be an intermediate stage in the morphological transformation of field spirals into cluster S0s.
\end{abstract}

Key words. galaxies: clusters: general - galaxies: clusters: individual: Abell 3921 - galaxies: distances and redshifts galaxies: evolution - cosmology: observations

\section{Introduction}

Over the past 30 years, observations of galaxies in different environments have confirmed a connection between galaxy properties and the density of the environment; for instance, at present a correlation between galaxy morphology and galaxy number density (Dressler 1980) is well established. Galaxies are found in environments of different density: from $0.2 \rho_{0}$ in voids up to $100 \rho_{0}$ in the inner regions of galaxy clusters (Boselli \& Gavazzi 2006), where $\rho_{0}$ is the average field density (Geller \& Huchra 1989). Since massive galaxy clusters are still growing in the present epoch (e.g. Donnelly et al. 2001), they provide a tool for testing hypotheses on the processes driving the evolution of galaxies. In this context, merging cluster systems are of special interest because they not only represent high-density environments but can also show complex dynamics, increased intra-cluster medium (ICM) turbulence, and shock waves (e.g. Markevitch et al. 2002; Paul et al. 2011; Roettiger et al. 1996).

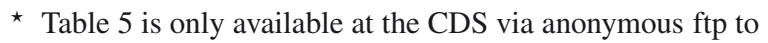
cdsarc.u-strasbg. fr (130.79.128.5) or via http://cdsarc.u-strasbg.fr/viz-bin/qcat?J/A+A/557/A62
By studying galaxy properties and their evolution with time (and galaxy number density), different interaction processes have been distinguished. Galaxy-galaxy interactions can lead to morphological distortions such as warps, bars, or tidal tails due to close spatial encounters of two (or more) galaxies. They are most efficient at low relative velocities, i.e. in groups or pairs of galaxies or in the outer regions of galaxy clusters (e.g. Toomre $\&$ Toomre 1972). In the latter case, galaxy-galaxy interactions can induce increased star formation rates (SFR; e.g. Porter \& Raychaudhury 2007) before the galaxies have been stripped of their gas during the process of falling towards the denser core regions of the cluster (see below).

Galaxy harassment (Moore et al. 1996) occurs in galaxy clusters when a galaxy is exposed to tidal forces due to the cluster's gravitational potential and consecutive fly-bys with other cluster members in the core regions. This process can affect many properties of a galaxy within a cluster, including the gas distribution and content, the orbital distribution of stars, and the overall shape.

Furthermore, hydrodynamic interactions between the gaseous component of a galaxy and the hot gas trapped in the 
cluster's potential have been investigated via observations and simulations, such as ram pressure stripping (e.g. Abadi et al. 1999) or galaxy strangulation (e.g. McGee et al. 2009). In the former case the movement of the infalling galaxy relative to the ICM causes ram pressure that can (partially) remove the galaxy's gaseous halo and disk. This process can first enhance (e.g. Kapferer et al. 2009) and eventually quench (e.g. Quilis et al. 2000) star formation, depending on density, velocity and timescale.

The gaseous halo represents the hot phase of a galaxy's gas distribution that is gravitationally less bound than the cold gas disk. It is thus more easily affected by ICM interaction. This process can be even more efficient when a galaxy experiences efficient (i.e. low-velocity) tidal interactions with other cluster members in the outer regions of a cluster (e.g. Balogh et al. 2000). After removal of the gaseous halo the only internal gas reservoir is the gas disk which eventually is consumed via star formation - the galaxy gets "strangulated". This process is characterised by a gradual decline of the SFR on relatively long timescales (e.g. Berrier et al. 2009).

Although at different rates, galaxy-galaxy interactions and ram pressure stripping have been shown to occur also in galaxy subclusters and galaxy groups (e.g. Fujita 2004), thereby preprocessing the galaxies with respect to a future cluster infall. Compared to other environments, (compact) groups of galaxies have been found to show the highest fraction of morphologically distorted galaxies (e.g. Mendes de Oliveira \& Hickson 1994).

Merger-induced shocks in the ICM have been observed for more than ten years (e.g. Markevitch et al. 2002). These shocks lead to strong increases in temperature and density in the ICM. It is still a matter of debate whether ICM shocks could increase the efficiency of ram pressure stripping and thereby have an influence on SFRs and galaxy morphology even in the intermediate density regimes of galaxy cluster infall regions. However, ram pressure stripping has been observed to be efficient even in the outskirts of galaxy clusters up to clustercentric distances of several virial radii (e.g. Kantharia et al. 2008).

In this paper we present a spectroscopical and, for the first time, morphological analysis of the galaxies in the merging cluster Abell 3921.

Abell 3921 is classified as an Abell cluster of richness 2 and BM-type II at $z \simeq 0.093$. Follow-up XMM-Newton/EPIC observations (Belsole et al. 2005) confirmed a bimodal galaxy distribution and, together with numerical simulations (Kapferer et al. 2006), led to the conclusion that Abell 3921 represents an offaxis merger between a main cluster and a less massive subcluster falling in from the south. Combined optical and X-ray studies have established a mass-ratio of 5:1 (Ferrari et al. 2005).

Throughout this paper we assume $H_{0}=70 \mathrm{~km} \mathrm{~s}^{-1} / \mathrm{Mpc}$, $\Omega_{\mathrm{m}}=0.3, \Omega_{\Lambda}=0.7$. At the systemic cluster redshift of 0.093 , 1 arcmin corresponds to $\sim 104 \mathrm{kpc}$ in this cosmology.

\section{The data}

Multifibre spectroscopical service observations in the central $2 \times$ $2 \mathrm{deg}^{2}$ of A3921 were carried out using the two-degree field (2dF) system on the AAT (Lewis et al. 2002). We used the 400 fibre positioner and double spectrograph system of $2 \mathrm{dF}$ to perform two sets of observations both centred at $\alpha=22^{\mathrm{h}} 49^{\mathrm{m}} 45.84^{\mathrm{s}}$ $\delta=-64^{\circ} 22^{\mathrm{m}} 16.32^{\mathrm{s}}$. Fibre allocation was carried out using the $2 \mathrm{dF}$ Configure programme, which optimises fibre placement based on user-defined weights associated to each target present in the input catalogue and on a set of instrumental limitations (e.g. minimum distance between allocated fibres). The required input file was created using the catalogue derived from our deep imaging observations $(V, R$, and $I$ passbands, mean seeing: $1.25^{\prime \prime}$, magnitude limit: 22.7 for $R$ band imaging used in this paper; for details see Ferrari et al. 2005). It included a list of fiducial stars with $R_{A B}=13.7-14.7$, blank sky positions and programme sources, i.e. objects classified as galaxies in our imaging catalogue. The highest weight (i.e. priority) was given to galaxies brighter than $R_{A B}=18.5$ in order to reach the highest possible completeness level at this magnitude cut $\left(R_{A B}^{*}+2.1\right)$.

We used the same $2 \mathrm{dF}$ grating (i.e. $300 \mathrm{~B}$ ) in the two available spectrographs of $2 \mathrm{dF}$ and we adopted a central wavelength of $6000 \AA$. Our observations thus gave 200 spectra per observing run and spectrograph with a spectral resolution of $8-9 \AA$ and covered the approximate wavelength range 3800-8200 $\AA$ which includes all spectral features from [OII] $3727 \AA$ to $\mathrm{H} \alpha 6563 \AA$ at A3921 redshift $(z \approx 0.093)$. Two runs of observations were carried out in October 2004 and November 2004 with a total exposure time of $3600 \mathrm{~s}$ per run, divided in three exposures of $1200 \mathrm{~s}$ in order to eliminate cosmic rays. The data were reduced at the telescope using the $2 \mathrm{dF}$ data reduction pipeline software $2 \mathrm{dfdr}$.

\subsection{Redshift determination}

We determined redshifts using both cross correlation against template spectra (Tonry \& Davis 1979) and identification of possible emission lines. We first used the automatic redshift code for $2 \mathrm{dF}$ spectra, runz (Colless et al. 2001). Then, in order to do a visual check of every best automatic redshift, we measured radial velocities with the IRAF task xcsao ${ }^{1}$ of the RVSAO package. In this second step, the results of runz were adopted as initial guess for radial velocities. In both cases, we used as radial velocity standards the eight templates adopted by Colless et al. (2001), which include five galaxies and three stars covering a broad range of spectral types. A good agreement between the two different measures was obtained, with a mean offset of $16.9 \mathrm{~km} \mathrm{~s}^{-1}$ and a standard deviation of $104.2 \mathrm{~km} \mathrm{~s}^{-1}$ for our high quality redshift catalogue (velocity flag (VF) $0 \mathrm{ob}-$ jects - see below). In the following, the measurements derived through the IRAF task xcsao and checked by eye are given and used.

A total of 597 redshift estimates have been obtained, among which 546 were derived from high signal-to-noise ratio $(\mathrm{S} / \mathrm{N})$ spectra and thus are very precise $(\mathrm{VF}=0), 24$ were obtained from lower $\mathrm{S} / \mathrm{N}$ spectra, which allowed a redshift measurement but with a higher uncertainty $(\mathrm{VF}=1)$, and 27 correspond to bad S/N spectra, not sufficient to get a reliable redshift estimate $(\mathrm{VF}=2)$. Among the 597 objects of the total catalogue, 74 are stars (67 with $\mathrm{VF}=0,6$ with $\mathrm{VF}=1$ and 1 with $\mathrm{VF}=2), 479$ are galaxies with very good $z$-determination $(\mathrm{VF}=0)$, while 18 and 26 are respectively the galaxies with an uncertain $(\mathrm{VF}=1)$ or bad $(\mathrm{VF}=2) z$ estimate. 61 objects of the catalogue were observed twice, the total number of non-blank sky spectra observed being 658. In all these cases, we kept the measurement characterised by the highest value of $R$, i.e. the parameter related to the $\mathrm{S} / \mathrm{N}$ of the correlation peak given by xcsao. In summary, $91.6 \%$ of the galaxy redshifts are of high quality, $3.4 \%$ are of low quality and for $5 \%$ it was not possible to get a reliable redshift estimate.

Among the 61 couples, the mean offset in velocity between the $\mathrm{VF}=0$ objects is $-24.8 \mathrm{~km} \mathrm{~s}^{-1}$ with a standard deviation of $97.0 \mathrm{~km} \mathrm{~s}^{-1}$. Based also on the previous comparison between xcsao and runz results, we therefore conclude

For emission line galaxies we also used the task emcsao. 
that radial velocities $(c z)$ are measured with a typical error of $\sim 100 \mathrm{~km} \mathrm{~s}^{-1}$. As in Barrena et al. (2007) the nominal errors given by xcsao show generally lower values, ranging between $10.5 \mathrm{~km} \mathrm{~s}^{-1}$ and $121.8 \mathrm{~km} \mathrm{~s}^{-1}$ with a mean uncertainty of $48.6 \mathrm{~km} \mathrm{~s}^{-1}$, a factor of $\sim 2$ lower than expected. In order to further test this result, we estimated the ratio between a) the difference in the radial velocities measured with xcsao and runz for the VF $=0$ objects; and $b$ ) the corresponding nominal xcsao errors, obtaining a mean value of 1.8. In the following analysis we therefore multiply the nominal xcsao errors by a factor of 2 .

A3921 has been the object of previous spectroscopical observations (da Costa et al. 1991; Dalton et al. 1994; Ferrari et al. 2005; Katgert et al. 1996, 1998; Mathewson \& Ford 1996; Muriel et al. 2002; Pimbblet et al. 2006; Way et al. 2005). It is important to stress that all previous observations are however characterised by a lower spectral coverage ( 3500-6800 $)$.

Optical imaging has been taken with the Wide Field Imager camera at the ESO $2.2 \mathrm{~m}$ telescope at La Silla observatory. For details see Ferrari et al. (2005, Sect. 2.1).

\section{Redshift distribution and cluster membership}

The redshift distribution of the $479 \mathrm{VF}=0$ galaxies in the $2 \times 2$ square degrees field centred on A3921 is shown in Fig. 1 . In agreement with previous results (Ferrari et al. 2005; Pimbblet et al. 2006), the bulk of the cluster is clearly visible as a concentration between $z \sim 0.082$ and $z \sim 0.102$. In order to identify cluster members, we applied the standard iterative $3 \sigma$ clipping (Yahil \& Vidal 1977) as well as a modified $3 \sigma$ clipping involving the biweight estimators for location and scale (Beers et al. 1990) to an initial galaxy sub-sample with redshifts in a wider range $(0.075 \leq z \leq 0.110)$. Both versions of the $3 \sigma$ clip reduced the sample from 231 to 223 objects in the redshift range $0.0841 \leq z \leq 0.1006$. All these objects also satisfy the technique based on gaps in the velocity histogram proposed by Zabludoff et al. (1993) to filter out foreground and background galaxies. In agreement with the results of previous $2 \mathrm{dF}$ observations of A3921 (Pimbblet et al. 2006), these two simple methods, which make use of only one parameter (the apparent radial velocity of galaxies), indicate as cluster members those galaxies (223) in the redshift range $0.082 \leq z \leq 0.102$.

\subsection{Cluster substructure}

Earlier investigations (Belsole et al. 2005; Ferrari et al. 2004, 2005, 2006) identify A3921 as a merging system that consists of two galaxy clusters (A3921-A, A3921-B; mass ratio 5:1), each containing a giant elliptical galaxy (BCG1, BCG2) coincident with the respective peaks in X-ray emission (Belsole et al. 2005; Ferrari et al. 2004) within $\sim 22$ arcsec (corresponding to $\sim 38 \mathrm{kpc}$ at the cluster redshift) on average. To further investigate the substructure of A3921 on the basis of our new 2dF data we applied the Dressler-Shectman (DS-)test (Dressler \& Shectman 1988) to our sample of 223 confirmed cluster galaxies as a whole. An illustration of this test's outcome is given in Fig. 2.

The DS-test reveals some 3D-substructure (significance value $P<5 \times 10^{-5}$ for $10^{6}$ Monte-Carlo iterations; this corresponds to $>99.99 \%$ ) in the south-east of the cluster region between $22^{\mathrm{h}} 51^{\mathrm{m}}$ and $22^{\mathrm{h}} 56^{\mathrm{m}}$ in right ascension and $-64^{\circ} 24^{\mathrm{m}}$ and $-65^{\circ} 10^{\mathrm{m}}$ in declination. The newly detected clump of galaxies is further referred to as "component C" or "A3921-C". We assigned 16 galaxies to component $\mathrm{C}$ using a combined positionredshift space criterion. We first isolated all 37 galaxies within a

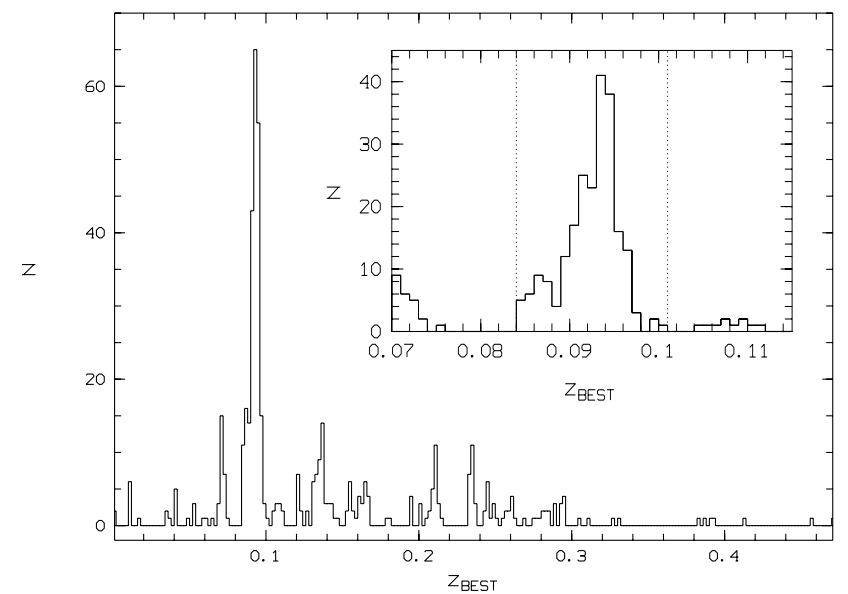

Fig. 1. Redshift histogram of the $479 \mathrm{VF}=0$ galaxies in the $2 \times 2$ square degrees field centred on A3921 with a binning of $\Delta z=0.002$. Inlay: redshift histogram of the $\mathrm{VF}=0$ galaxies in the redshift range $0.0841 \leq z \leq 0.1006$ with a binning of $\Delta z=0.001$. The dashed lines indicate the redshift limits for cluster members derived via the $3 \sigma$ clipping technique (see text for details).

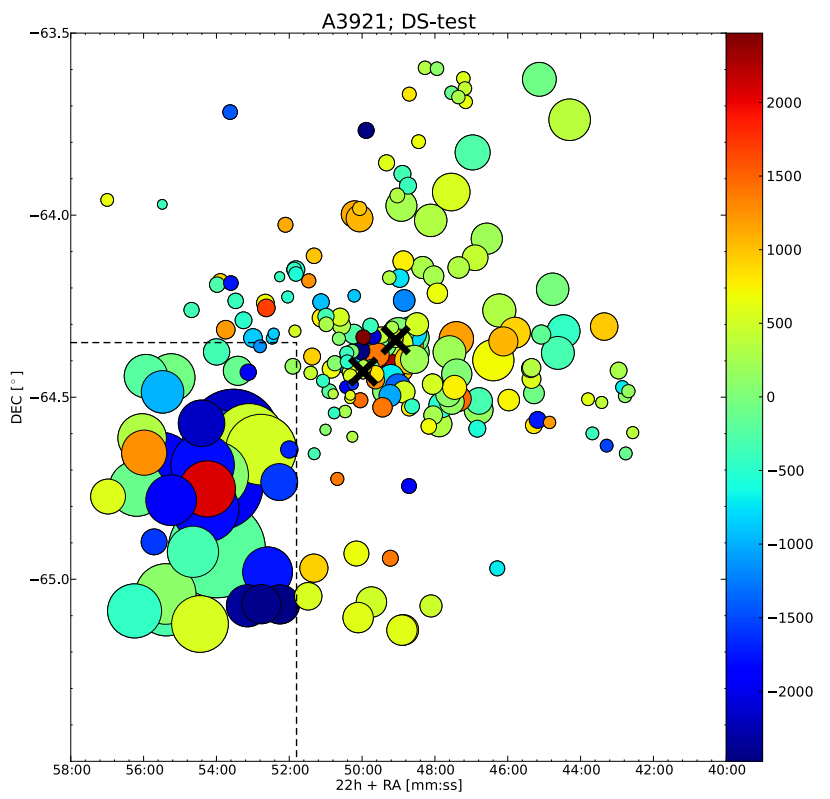

Fig. 2. Graphical representation of the DS-test output. The plot shows the spatial location of the galaxies. The radii of the plotted circles are proportional to $e^{\delta}$ where $\delta$ is the DS-test measure of the local deviation from the global velocity dispersion and mean recessional velocity, i.e. larger symbols correspond to a higher significance of residing in a substructure. The colours indicate the rest-frame velocity relative to the cluster centre (BCG1) in $\mathrm{km} \mathrm{s}^{-1}$. BCG1 and BCG2 are marked by black crosses, respectively. Obviously there is some substructure detected in the south-east region of the cluster (indicated by the dashed lines). For a comparison with subcluster membership see Fig. 5.

projected rectangular region confined to the south and east by the borders of our field of view and to the north and west by the lines of longitude at $22^{\mathrm{h}} 51^{\mathrm{m}} 48^{\mathrm{s}}$ and latitude at $-64^{\circ} 21^{\mathrm{m}}$, respectively (see Fig. 2), thereby keeping all galaxies with $\delta \geq 3$ (see caption of Fig. 2). Second, motivated by the redshift distribution shown in Fig. 3, we introduced a limit of $z=0.0875$ in redshift space. All 16 galaxies with redshifts lower than 0.0875 were assigned to A3921-C. The two galaxies at $z=0.0891$ and $z=0.0885$ were considered ambiguous members of component $\mathrm{C}$ according to 


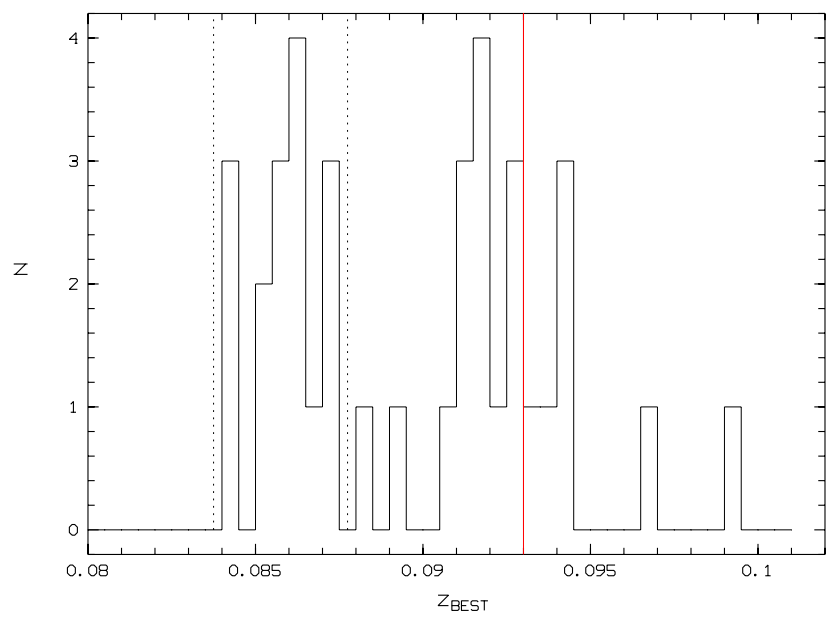

Fig. 3. Distribution of the 37 galaxies within the rectangular segment indicated in Fig. 2 in redshift space. The dashed vertical lines confine the range of objects assigned to component $\mathrm{C}$. The red solid line refers to the median cluster redshift of $z=0.093$. The two galaxies between $z=0.088$ and $z=0.090$ were considered ambiguous members of component $\mathrm{C}$ and therefore discarded.

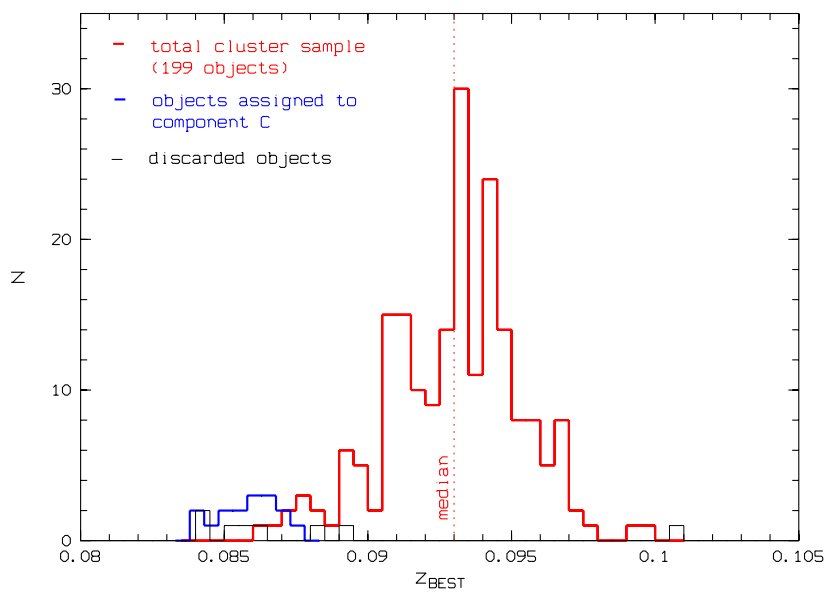

Fig. 4. Redshift distribution of the final cluster sample. Red: adjusted total sample of A3921 in z-space. Blue: objects assigned to component C. Black thin line: objects clipped after removal of component $\mathrm{C}$. The two galaxies around $z=0.089$ are ambiguous members of component $\mathrm{C}$. The median redshift of the adjusted total cluster sample is indicated by the dashed vertical line.

their intermediate position in $z$-space and therefore discarded. Reapplying the $3 \sigma$ clippings on the remaining sample of 205 galaxies reduces the number of cluster members to 199. For the separation of this sample into A3921-A and A3921-B we simply measure the projected distance of each galaxy to BCG1 and BCG2, respectively. We do not have any other means for separation due to the similar redshift distributions of components A3921-A and A3921-B. The redshift histogram in Fig. 4 illustrates the $z$-distribution of our final cluster sample as well as the rejected objects or those assigned to component $\mathrm{C}$, respectively. Follow-up DS-tests on the subsamples show significance values of $P=0.19$ and $P=0.59$ for A3921-A and A3921-B, respectively, using $10^{6}$ Monte-Carlo iterations. These values are above the generally adopted threshold of $P=0.1$. The DS-test hence confirms that the redefined cluster samples of A3921-A and A3921-B do not contain substructure.

Reapplying the $3 \sigma$ clippings to the cluster subsamples reduces the galaxy number in A3921-B by 4 whereas A3921-A

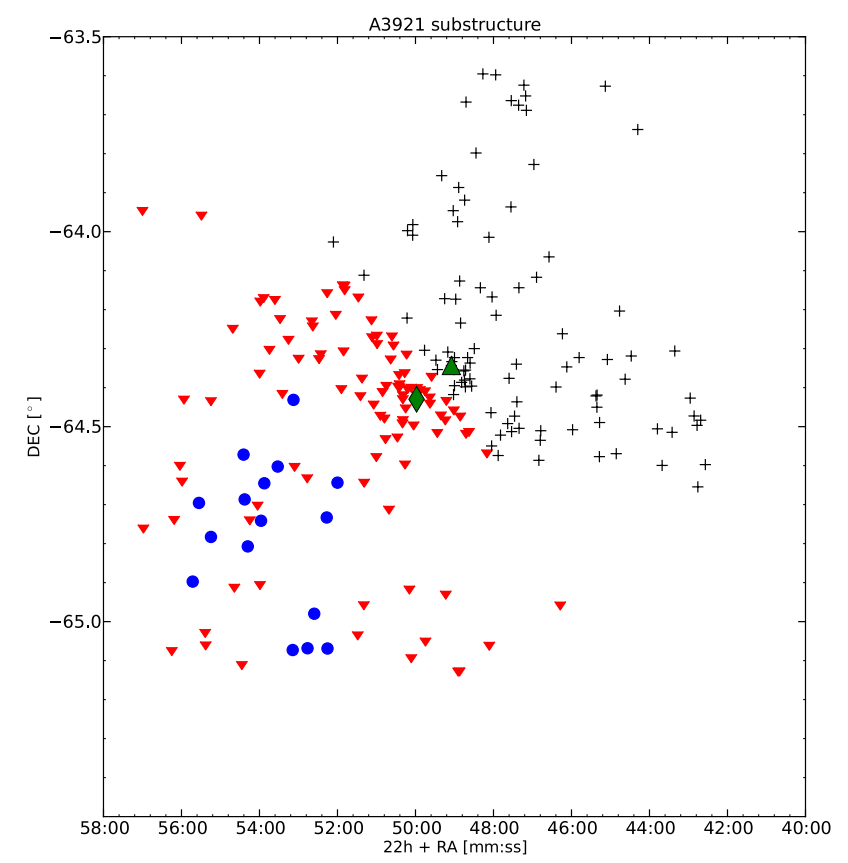

Fig. 5. Substructure of A3921. Red triangles indicate objects belonging to subcluster A, black crosses represent galaxies in subcluster B and blue circles show objects assigned to component $\mathrm{C}$. BCG1 and BCG2 are indicated by a green diamond and a green triangle, respectively.

is not affected. In the end we have 101 galaxies assigned to A3921-A and 94 galaxies within A3921-B. Figure 5 shows the spatial distribution of the subdivided sample, Fig. 6 illustrates the structure of A3921-A and A3921-B in redshift-space. Table 1 lists the median redshift and the biweight estimators for scale ( $S_{B I}$, corresponding to the velocity dispersion) as well as estimations for radius and total mass calculated on the assumption of isothermal spheres (Carlberg et al. 1997) and on the basis of velocity dispersion and 3D harmonic radius (Small et al. 1998). The sum of the masses of A3921-A and A3921-B clearly exceeds the mass estimate for the whole cluster. This is due to the estimation technique and reflects the lack of a physical separation criterion for subclusters $\mathrm{A}$ and $\mathrm{B}$. Note that the velocity dispersion is slightly lower for the whole sample than for A3921-A in compliance with previous results by Ferrari et al. (2005). This surprising result can be explained by the individual redshift distributions of components $\mathrm{A}$ and $\mathrm{B}$ which have a similar width but are differently skewed. Consequently the estimate for $r_{200}$ is slightly lower for the whole sample than for A3921-A. The respective values are, however, compatible within the error. Also note that our estimates for $r_{200}$ of A3921-A and A3921-B are larger by a factor of $\sim 4$ than the results of Ferrari et al. (2005). The mass estimates for A3921-A and A3921-B by Ferrari et al. (2005) are lower than ours by factors of $\sim 4$ and $\sim 10$, respectively. Note that the mass estimate for A3921-A by Ferrari et al. (2005) based on velocity dispersion is in agreement with the X-ray mass estimate by Belsole et al. (2005). Our estimate for $r_{200}$ of A3921-(A+B) is in excellent agreement with an estimate using the caustics method (Diaferio 1999) on galaxies between $23000 \mathrm{~km} \mathrm{~s}^{-1}$ and $33000 \mathrm{~km} \mathrm{~s}^{-1}$ in $c z$-space, where $r_{200}=1.62 \mathrm{Mpc}$ is found. The same technique yields a mass estimate of $1.28 \times 10^{14} M_{\odot}$ for A3921-(A+B) which differs from our result. However, note that our computations are based on a virialised system which is probably not fulfilled. Hence, cluster masses might be overestimated in our approach. 
Table 1. Velocity dispersion $\left(S_{B I}\right), r_{200}$ and $M$ for A3921-A, A3921-B and the total sample.

\begin{tabular}{lccccc}
\hline \hline Subsample & $N_{\text {gal }}$ & $\langle z\rangle$ & $\begin{array}{c}S_{B I} \\
{[\mathrm{~km} / \mathrm{s}]}\end{array}$ & $\begin{array}{c}r_{200} \\
{[\mathrm{kpc}]}\end{array}$ & $\begin{array}{c}M \\
{\left[10^{15} M_{\odot}\right]}\end{array}$ \\
\hline Whole sample & 199 & 0.0932 & $687_{-65}^{+51}$ & $1627_{-154}^{+121}$ & $2.03_{-0.06}^{+0.07}$ \\
A3921-A & 101 & 0.0929 & $754_{-89}^{+66}$ & $1786_{-211}^{+156}$ & $1.73_{-0.12}^{+0.13}$ \\
A3921-B & 94 & 0.0934 & $529_{-71}^{+50}$ & $1253_{-168}^{+118}$ & $0.91_{-0.06}^{+0.06}$ \\
Component C & 16 & 0.0860 & & & \\
\hline
\end{tabular}

Notes. Additionally the number $N_{\text {gal }}$ of $3 \sigma$-member galaxies and the median redshift are given for both subclusters, the total sample and component C. We do not attempt to compute $S_{B I}, r_{200}$ or $M$ for component $\mathrm{C}$ due to the small number of identified members.

The caustics method (for details see Diaferio 1999; Serra et al. 2011) is an approach to determine cluster membership and estimate mass even for the outskirts of galaxy clusters at distances up to several Mpc from the cluster centre. In such regions the usual assumption of virialisation does not hold and mass estimates relying on this assumption become inaccurate. Caustics are the boundaries of a trumpet-shaped area populated by cluster galaxies in the redshift space diagram (projected clustercentric distance vs. redshift). The caustics method is based only on redshifts and positions. At each distance from the cluster centre it relates the width of the trumpet-shape in redshift to the escape velocity of a given cluster. Masses are estimated by assuming that clusters are spherically symmetric and form by hierarchical clustering.

As a result of the analysis of our new $2 \mathrm{dF}$ data, the mass ratio $R_{M}$ between $\mathrm{A} 3921-\mathrm{A}$ and $\mathrm{A} 3921-\mathrm{B}$ (excluding component C) becomes $R_{M}=1.90_{-0.01}^{+0.02}$ whereas Ferrari et al. (2005) found $R_{M} \sim 5$.

A numerical simulation of the A3921 system based on a mass ratio of $\sim 5$ and including galactic winds and star bursts (Kapferer et al. 2006) suggests that the merging process of A3921-A and A3921-B is in a phase where the closest encounter has not happened yet.

The caustics method has also been used to determine cluster membership based on a galaxy catalogue of A3921 containing 372 galaxies including the objects from our dataset in the $c z$-range between $23000 \mathrm{~km} \mathrm{~s}^{-1}$ and $33000 \mathrm{~km} \mathrm{~s}^{-1}$ (Serra \& Diaferio 2013). Comparing the resulting sample of 195 galaxies with A3921- $(\mathrm{A}+\mathrm{B}+\mathrm{C})$ which consists of 215 objects, 119 galaxies were identified as cluster members. Note that all galaxies assigned to A3921-C are among the non-cluster members. The redshift distribution of these 96 non-cluster member objects ranges from $z=0.084$ to $z=0.100$ and is characterised by a median value of 0.094 as well as a mean of 0.093 with a standard deviation of 0.003 .

Since the main science driver of this paper is not the identification of the virialised cluster galaxies, but the impact of the complex environment of A3921 on the galaxy properties, we will in the following use the definition of cluster membership presented earlier in this section.

\subsection{Field sample definition}

Initially our field sample consists of 256 galaxies left over after deploying the aforementioned limitation procedures on the total galaxy sample, i.e. we neither include the objects supposedly belonging to A3921-C nor objects being ruled out by individual

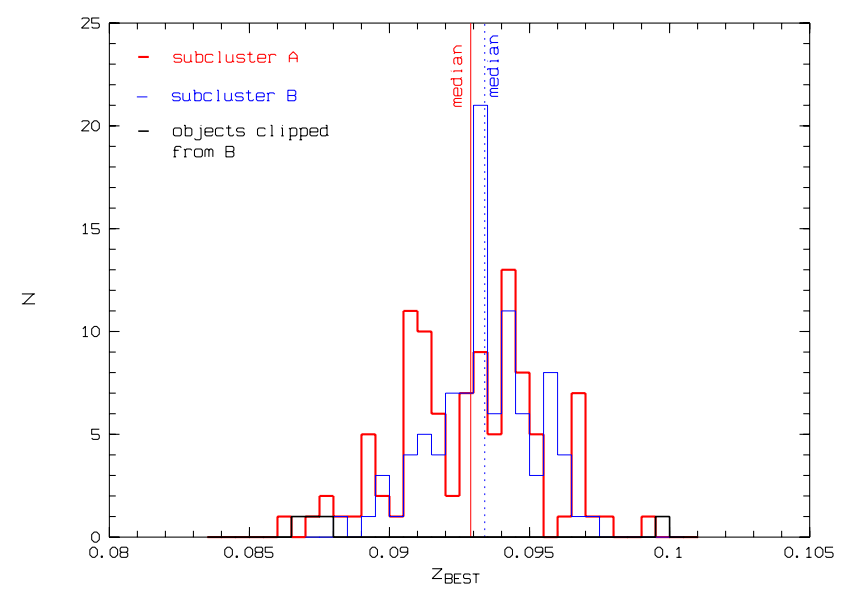

Fig. 6. Structure of subclusters A and B in z-space. The black line shows 4 objects clipped from the B-sample. The median redshifts of the samples are indicated by the thin red and the dashed blue line, respectively.

$3 \sigma$ clippings on the subsamples A3921-A and A3921-B. The initial field sample strongly differs from the cluster sample in its redshift- and magnitude distributions. To allow a fair comparison, we choose upper and lower redshift limits on the field sample such that the field and cluster sample have similar luminosity distribution and the same mean redshift. Thereby the field sample gets reduced to 83 galaxies. This basic field sample is being used whenever we present comparisons between the field and either the whole cluster (A3921-A + A3921-B) or one of the subclusters, respectively. All further subdivisions applied to the cluster sample analogously apply to the basic field sample. For our morphological analysis the field sample reduces to 74 galaxies due to quality lacks in the respective optical images. This sample will be referred to as the morphological field sample.

\section{Spectroscopical analysis}

\subsection{OII and $\mathrm{H} \alpha_{\alpha}$ equivalent width measurement}

We determined emission line equivalent widths (EWs) from our $2 \mathrm{dF}$ spectra in the following way: The continuum signal was measured within two intervals of $10 \AA$ width $20 \AA$ left and right from a given emission line, respectively. Next a Gaussian fit was applied to the emission line (in the case of the [OII]-doublet we used a double Gaussian fit with equal FWHM). This fit, as well as the final computation of the EW, was based on the assumption that the continuum intensity at the position of the emission line was the mean of the continuum intensity in the two windows left and right of the emission line.

For eight objects with low $\mathrm{S} / \mathrm{N}$, the [OII] line could not be fitted with a doublet but only with a single line profile. We corrected the [OII] EWs of these galaxies with a factor of 1.25 determined from 25 objects with the highest $\mathrm{S} / \mathrm{N}$, for which we compared robust doublet fits to singlet fits.

We estimated the EW upper limits of [OII] and $\mathrm{H} \alpha$ by simulating spectra with lines of a FWHM of $4.3 \AA$ (our dispersion) and continuum fluxes and noise from spectra for which we obtained the lowest EW values. This leads to two different estimates for [OII] and $\mathrm{H} \alpha$. We simulated EWs between $0.25 \AA$ and $25 \AA$. Requiring that input and output EW had to agree within the measurement errors, we thus found a minimum EW of $4.9 \AA$ for [OII] and $1.5 \AA$ for $\mathrm{H} \alpha$. 


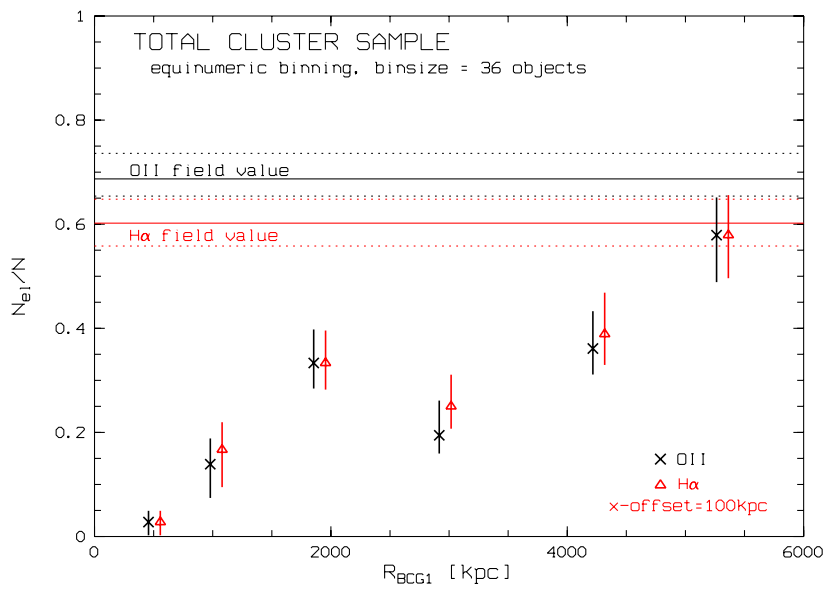

Fig. 7. Fraction of galaxies showing [OII] or $\mathrm{H} \alpha$ emission lines, respectively, vs. distance from the cluster centre. The corresponding data for the subsamples of components A and B are listed in Table 2.

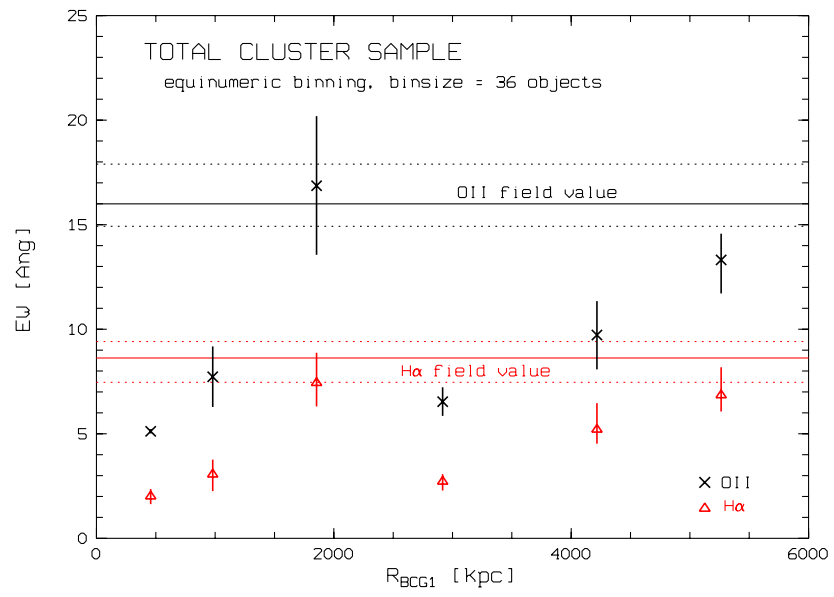

Fig. 8. Mean equivalent width of $[\mathrm{OII}]$ and $\mathrm{H} \alpha$ emission lines for all objects (objects without detectable lines get assigned a lower threshold value). The corresponding data for the subsamples of components A and $\mathrm{B}$ are listed in Table 2.

\subsection{Equivalent widths and star formation rates}

Figures 7 to 10 show the projected radial trends (with respect to the centre of A3921-A and of the whole sample taken to be coincident with BCG1) of

a) the fraction of EL-galaxies (i.e. galaxies definitively showing [OII], $\mathrm{H} \alpha$ or both emission lines) per bin;

b) the equivalent width of [OII $]$ and $\mathrm{H} \alpha$ lines and

c) [OII]-based star formation estimates

for the total sample (i.e. A3921-(A+B)). The corresponding data for both subsamples are given in Tables 2 and 3. To estimate the SFR of galaxies we adopt a relation presented in Kennicutt (1992b):

$$
\operatorname{SFR}\left(M_{\odot} \mathrm{yr}^{-1}\right) \simeq 2.7 \times 10^{-12} \frac{L_{R}}{L_{R}(\odot)} E W([\mathrm{OII}]) E(\mathrm{H} \alpha)
$$

where $E W([\mathrm{OII}])$ denotes the $z$-corrected equivalent width of [OII] and $E(\mathrm{H} \alpha)$ stands for the extinction value at the respective wavelength. To apply this measure to our data we first had to determine the $R$-band absolute magnitudes $L_{R}$. For this purpose we used the image processing software SExtractor (Bertin \& Arnouts 1996) calibrated via magnitudes of 37 objects we could identify in the magnitude catalogue appended to

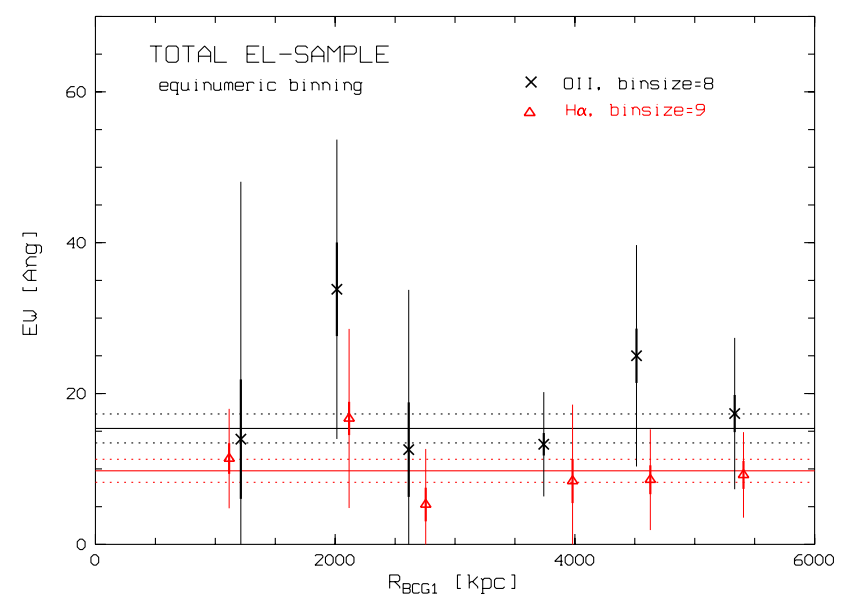

Fig. 9. Median equivalent width of [OII] and $\mathrm{H} \alpha$ emission lines only for EL-objects. The thin error bars represent the standard deviation within each data bin. The corresponding data for the subsamples of components $\mathrm{A}$ and $\mathrm{B}$ are listed in Table 3 . The horizontal lines represent the field values and $1 \sigma$ errors.

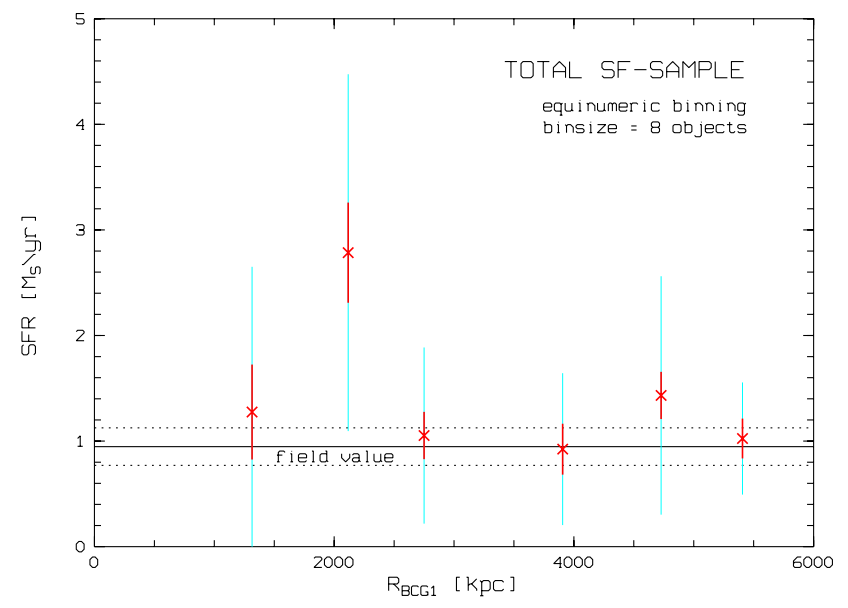

Fig. 10. Median star formation rate for objects with detectable [OII] emission line. The thin error bars represent the standard deviation within each data bin. The corresponding data for the subsamples of components A and B are listed in Table 3.

Ferrari et al. (2005). Moreover, for a proper k-correction all spectra were classified by comparison with spectra from Kennicutt (1992a).

All errors given in Figs. 7 to 10 and Figs. 13 to 15 as well as in Tables 2-4 are generated via bootstrapping techniques except for bins with less than ten objects where the standard deviation error is additionally given (indicated by thin error-lines in the plots). All clustercentric distances $\left(R_{\mathrm{BCG} 1}\right)$ are median values for each bin. To rule out potential binning biases we analysed different binning methods (equidistant and equinumeric binning) and a wide range of binsizes. The plots shown here reflect trends that are robust against binning changes. We use an equinumeric binning in these plots, i.e. whenever we show a given quantity as a function of radius, we keep the number of galaxies per bin fixed throughout the plot, except for the last data point which contains the respective remainder according to the total number of objects within the sample under investigation. Horizontal lines indicate the respective field values. Figure 7 illustrates fractions of galaxies showing emission lines. For the first EW-plot (i.e. Fig. 8), we assigned an equivalent width of $5.1 \AA$ to objects without a detectable [OII] doublet, and an EW of $1.6 \AA$ to the objects without 
Table 2. Mean equivalent widths and fractions of EL-galaxies (based on [OII] and $\mathrm{H} \alpha$, respectively) for subclusters A and B with a binsize of 20 objects.

\begin{tabular}{|c|c|c|c|c|c|c|c|c|c|}
\hline \multicolumn{5}{|c|}{ Subcluster A } & \multicolumn{5}{|c|}{ Subcluster B } \\
\hline $\begin{array}{l}R_{\mathrm{BCG} 1} \\
{[\mathrm{kpc}]}\end{array}$ & $N_{[\text {OII }]} / N$ & $N_{\mathrm{H} \alpha} / N$ & $\begin{array}{c}E W([\mathrm{OII}]) \\
{[\mathrm{Ang}]}\end{array}$ & $\begin{array}{c}E W(\mathrm{H} \alpha) \\
{[\mathrm{Ang}]}\end{array}$ & $\begin{array}{l}R_{\mathrm{BCG} 1} \\
{[\mathrm{kpc}]}\end{array}$ & $N_{\text {[OII }]} / N$ & $N_{\mathrm{H} \alpha} / N$ & $\begin{array}{c}E W([\mathrm{OII}]) \\
{[\text { Ang }]}\end{array}$ & $\begin{array}{c}E W(\mathrm{H} \alpha) \\
{[\mathrm{Ang}]}\end{array}$ \\
\hline 261.6 & 0 & 0 & 5.0 & 1.6 & 881.5 & $0.100_{-0.035}^{+0.025}$ & $0.150_{-0.085}^{+0.055}$ & $8.9_{-2.7}^{+1.7}$ & $2.0_{-0.2}^{+0.2}$ \\
\hline 713.2 & $0.100_{-0.030}^{+0.020}$ & $0.100_{-0.042}^{+0.054}$ & $5.6_{-0.3}^{+0.5}$ & $3.2_{-0.9}^{+1.1}$ & 1725.3 & $0.400_{-0.085}^{+0.055}$ & $0.400_{-0.073}^{+0.087}$ & $12.7_{-2.6}^{+3.6}$ & $7.6_{-1.7}^{+0.9}$ \\
\hline 1497.3 & $0.250_{-0.096}^{+0.096}$ & $0.250_{-0.040}^{+0.068}$ & $18.2_{-5.2}^{+6.2}$ & $6.5_{-2.5}^{+1.3}$ & 2788.9 & $0.200_{-0.040}^{+0.076}$ & $0.200_{-0.078}^{+0.096}$ & $5.5_{-0.3}^{+0.3}$ & $2.5_{-0.3}^{+0.5}$ \\
\hline 2719.9 & $0.200_{-0.050}^{+0.050}$ & $0.250_{-0.072}^{+0.110}$ & $8.3_{-1.6}^{+5.8}$ & $3.7_{-0.9}^{+1.3}$ & 3730.4 & $0.300_{-0.064}^{+0.088}$ & $0.350_{-0.083}^{+0.115}$ & $9.2_{-1.1}^{+2.5}$ & $4.9_{-1.2}^{+1.6}$ \\
\hline 4339.5 & $0.550_{-0.066}^{+0.052}$ & $0.600_{-0.077}^{+0.069}$ & $13.3_{-2.9}^{+2.3}$ & $7.2_{-1.7}^{+0.9}$ & 5101.7 & $0.429_{-0.107}^{+0.129}$ & $0.429_{-0.211}^{+0.209}$ & $10.1_{-1.8}^{+0.8}$ & $5.0_{-1.5}^{+1.5}$ \\
\hline
\end{tabular}

Notes. Field values (based on the whole field of view) are shown in Figs. 7 and 8.

Table 3. Median eqivalent widths and star formation rates in subclusters $\mathrm{A}$ and $\mathrm{B}$. Each radial bin contains 4 galaxies (5 for $E W_{\mathrm{H} \alpha}$, subcluster $\left.\mathrm{B}\right)$.

\begin{tabular}{|c|c|c|c|c|c|c|c|}
\hline \multicolumn{8}{|c|}{ Subcluster A } \\
\hline $\begin{array}{l}R_{\mathrm{BCG} 1} \\
{[\mathrm{kpc}]}\end{array}$ & $\begin{array}{c}E W([\mathrm{OII}]) \\
{[\mathrm{Ang}]}\end{array}$ & $\begin{array}{c}\mathrm{rms}_{E W(\mathrm{OII})} \\
{[\mathrm{Ang}]}\end{array}$ & $\begin{array}{c}\text { SFR } \\
{\left[M_{\odot} / \mathrm{yr}\right]}\end{array}$ & $\begin{array}{l}\mathrm{rms}_{(\mathrm{SFR})} \\
{\left[M_{\odot} / \mathrm{yr}\right]}\end{array}$ & $\begin{array}{l}R_{\mathrm{BCG} 1} \\
{[\mathrm{kpc}]}\end{array}$ & $\begin{array}{c}E W(\mathrm{H} \alpha) \\
{[\mathrm{Ang}]}\end{array}$ & $\begin{array}{c}\mathrm{rms}_{E W(\mathrm{H} \alpha)} \\
{[\mathrm{Ang}]}\end{array}$ \\
\hline 1204.0 & $17.1 \pm 1.6$ & 44.6 & $2.9 \pm 0.4$ & 1.4 & 1204.0 & $12.5 \pm 1.1$ & 5.8 \\
\hline 2130.7 & $48.7 \pm 3.2$ & 16.7 & $3.4 \pm 0.6$ & 1.6 & 2130.7 & $27.3 \pm 0.8$ & 11.6 \\
\hline 3035.5 & $16.6 \pm 1.8$ & 8.9 & $1.6 \pm 0.3$ & 0.8 & 2719.9 & $3.1 \pm 1.7$ & 6.8 \\
\hline 4208.6 & $15.0 \pm 1.9$ & 10.3 & $1.3 \pm 0.3$ & 0.9 & 4037.2 & $6.7 \pm 0.7$ & 4.0 \\
\hline 4896.0 & $24.3 \pm 3.6$ & 17.0 & $1.5 \pm 0.2$ & 0.7 & 4322.4 & $15.2 \pm 1.3$ & 4.9 \\
\hline 5540.7 & $25.3 \pm 10.2$ & 14.3 & $0.9 \pm 0.1$ & 0.8 & 5333.3 & $13.1 \pm 0.3$ & 4.2 \\
\hline \multicolumn{8}{|c|}{ Subcluster B } \\
\hline 1314.8 & $12.8 \pm 3.6$ & 9.1 & $0.9 \pm 0.6$ & 1.7 & 1115.7 & $6.0 \pm 1.9$ & 7.6 \\
\hline 1930.3 & $20.2 \pm 4.3$ & 13.8 & $1.4 \pm 0.2$ & 0.4 & 1744.5 & $12.7 \pm 0.3$ & 7.8 \\
\hline 2679.4 & $9.9 \pm 9.2$ & 30.5 & $0.8 \pm 0.3$ & 0.8 & 2816.1 & $6.4 \pm 3.6$ & 8.2 \\
\hline 3562.7 & $7.8 \pm 1.1$ & 3.6 & $0.7 \pm 0.1$ & 0.3 & 3648.4 & $8.8 \pm 0.8$ & 12.6 \\
\hline 4515.4 & $25.0 \pm 4.4$ & 12.6 & $1.4 \pm 0.2$ & 0.5 & 5101.0 & $4.4 \pm 3.0$ & 7.3 \\
\hline 5258.6 & $17.7 \pm 2.3$ & 5.6 & $1.1 \pm 0.5$ & 1.5 & 5869.9 & $6.9 \pm 3.6$ & 7.7 \\
\hline
\end{tabular}

Notes. Field values (based on the whole field of view) are shown in Figs. 9 and 10.

a detectable $\mathrm{H} \alpha$ line. These values correspond to the lowest measured EWs of [OII] and $\mathrm{H} \alpha$, respectively, and are justified by the mock spectra discussed at the end of the last section. For the second EW-plot (i.e. Fig. 9) only objects with detectable [OII] or $\mathrm{H} \alpha$ emission lines (constituting the EL-sample) were considered. For the SFR-plots (i.e. Fig. 10) only objects with detected [OII] emission (constituting the SF-sample) were considered.

It is noticeable that within a distance of about $r_{200}$ (for the case of A3921-B it is $r_{200}+d$ where $d \simeq 850 \mathrm{kpc}$ represents the projected distance between the BCGs) from BCG1 all investigated quantities show an increase towards larger clustercentric radii, in compliance with previous studies on the dependence of galaxy properties on environment (e.g. Verdugo et al. 2008). Furthermore, at a clustercentric distance of $\sim 3 \mathrm{Mpc}$, fractions of EL-galaxies as well as equivalent widths (see. Figs. 7 to 8 and Table 2) show a noticeable decline. Also the equivalent widths of EL-galaxies only and the SFR plots (see Figs. 9 to 10 and Table 3) show a decrease at these radii, albeit with worse number statistics.

Comparing both subclusters (especially at radial distances $>4 \mathrm{Mpc}$ ), the investigated quantities reach lower values in
A3921-B than in A3921-A, albeit at low statistical significance. However, due to the lack of a physical separation criterion between A3921-A and A3921-B and due to the low number statistics for SFR plots and the second series of emission line plots we do not attempt to further interpret this.

\section{Morphological analysis}

For our morphological analysis we use the WFI imaging data by Ferrari et al. (2005). From the combined sample of cluster and field galaxies $(199+83=282$ objects $)$ we discard 10 cluster objects and 9 field objects because of corrupted imaging and therefore end up with a total morphological sample of $263 \mathrm{spec}-$ troscopically confirmed galaxies. For each of these we calculate Gini coefficient, concentration index and $M_{20}$ index (Lotz et al. 2004) (the resolution of the WFI imaging is inefficient for an estimation of the asymmetry index, we therefore do not use this descriptor in the following). The Gini coefficient (G) was originally introduced to assess the distribution of wealth within a human population - here we use it to describe the distribution of pixel values in a galaxy's image (Abraham et al. 2003). It relates 


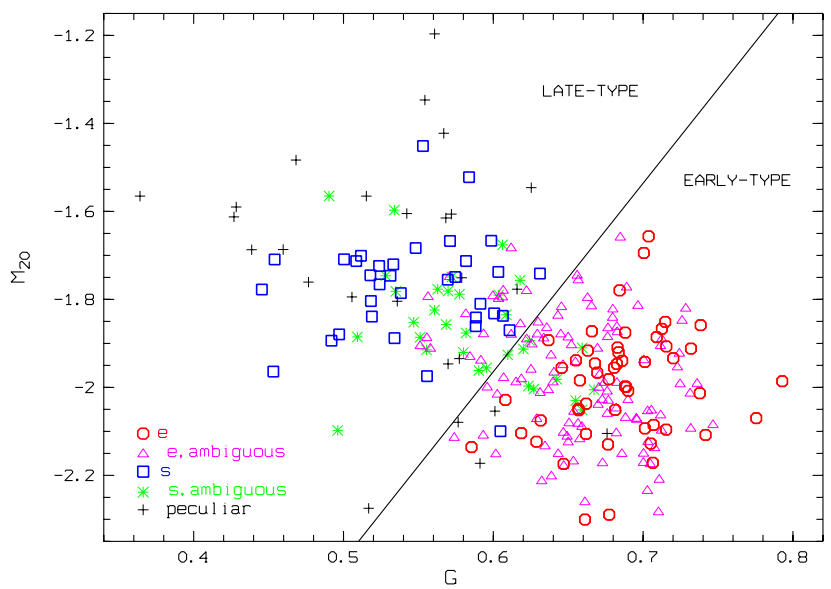

Fig. 11. Overall morphological sample (263 objects) in Gini- $M_{20}$ space, morphologically subdivided into spirals (s), ellipticals (e) and peculiars. The black line represents the cut applied to the unambiguously classified galaxies (blue squares and red circles) finally dividing the whole sample into late-type (upper left corner) and early-type galaxies (lower right corner). Objects classified as peculiar are included in the respective subsample. Note that we use this quantitative criterion throughout our morphological analysis.

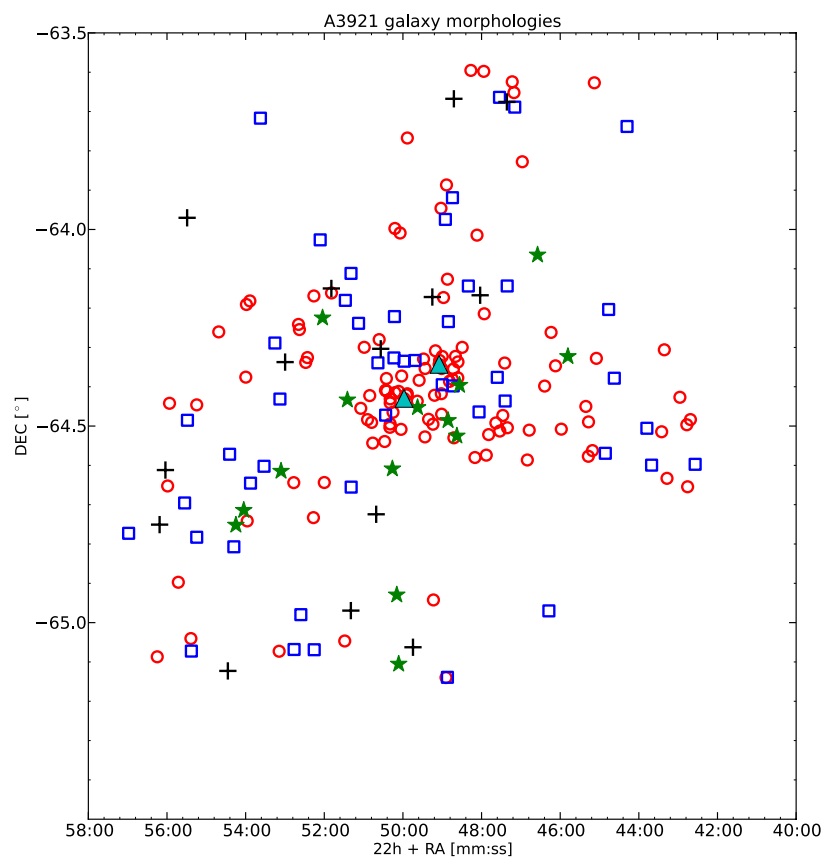

Fig. 12. Illustration of galaxy positions and morphological galaxy types. Early-type galaxies are shown as red circles, late-type objects are illustrated as blue squares. No-EL spirals are shown separately as green stars. Black crosses indicate morphologically distorted (i.e. peculiar) galaxies. BCG1 and BCG2 are represented by cyan triangles.

the Lorenz curve (Lorenz 1905) of a uniform flux distribution to the observed flux distribution. A Gini coefficient of zero corresponds to a completely uniform distribution while for a Gini coefficient of one, all the flux is contained within a single pixel. The concentration index $(\mathrm{C})$ measures the ratio of flux within an inner and an outer aperture. The $M_{20}$ index $\left(M_{20}\right)$ is defined as the second-order moment of the brightest 20 percent of the pixels in a galaxy image. This quantity hence is sensitive to bright off-centre features like star-forming regions.

As a start, we visually classify each galaxy as spiral, elliptical or peculiar (i.e. showing morphological distortions).

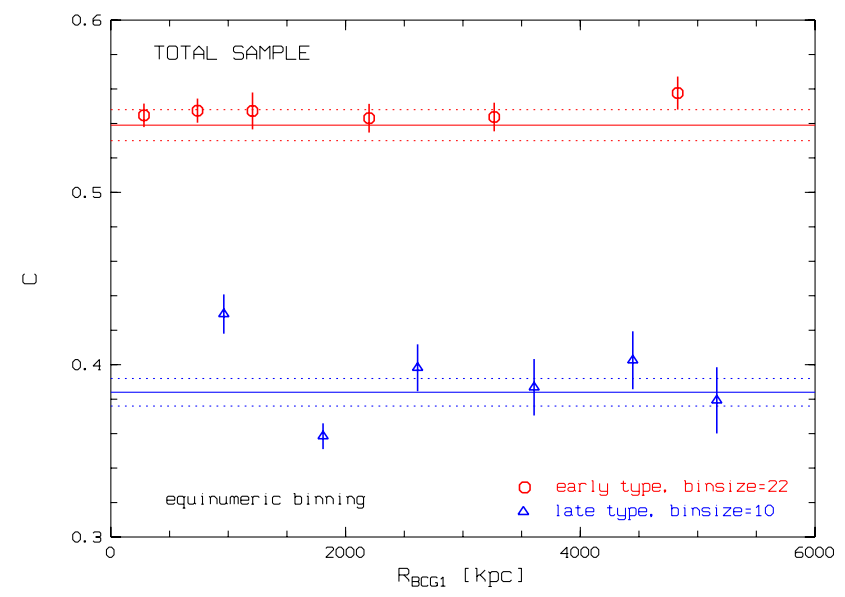

Fig. 13. Concentration index $\mathrm{C}$ as a function of clustercentric distance for early-type galaxies (red circles) and late-type galaxies (blue triangles). The corresponding data for the subsamples of components A and $\mathrm{B}$ are listed in Table 4 . The horizontal lines represent the field values and $1 \sigma$ errors.

Figure 11 shows the morphological sample (cluster and field) in Gini- $M_{20}$ space. Using only cases of unambiguous visual classifications (1/3 of our sample), we apply a dividing line in Gini$M_{20}$ space to separate between early-and late-type galaxies. This purely quantitative morphological classification will be used in the following. In Fig. 12 the positions and morphological types of all 189 members of the morphological cluster sample are depicted. Figures 13 to 15 illustrate the radial trends of the morphological quantities for the whole cluster sample. The corresponding data for components $\mathrm{A}$ and $\mathrm{B}$ are given in Table 4 (as before, the small number statistics do not allow component $C$ to be investigated separately). In the latter case as far as A3921-B is concerned the respective morphological subsample is reduced by 4 galaxies that were excluded from A3921-B by $3 \sigma$ clipping.

For the analyses of local surface number densities (see Sect. 6) we will at first add the objects assigned to component $\mathrm{C}$ to the morphological cluster sample and then make use of three different subsamples of this extended morphological sample (EMS): a spiral sample, a no-EL spiral sample and a peculiar sample. The spiral sample consists of all objects unambiguously classified as spirals as well as unclear cases located in the late-type regime of the Gini- $M_{20}$ space. We also define a no-EL spiral sample that holds morphologically classified spirals (i.e. galaxies identified as late-type either unambiguously by-eye or on the basis of their Gini/ $M_{20}$ indices) showing neither [OII] nor $\mathrm{H} \alpha$ emission line features. Note that the location in the calibrated Gini- $M_{20}$ space is a purely mathematical criterion which we use to classify all members of the morphological sample (field and cluster objects) either as a late-type or as an early-type galaxy. The peculiar sample consists of all galaxies visually identified as peculiar, i.e. showing morphological distortions (see Sect. 5.1). Objects with unambiguous deviations from an undisturbed morphology are classified as peculiar. However, as illustrated in Fig. 11 the peculiar sample shows a wide spread in Gini- $M_{20}$ space. It is hence impossible to define a quantitative Gini- $M_{20}$ criterion for peculiarity. This is due to the limited spatial resolution of our ground-based imaging. Thus, in contrast to the distinction into late-type and early-type galaxies, we keep a visual classification for the peculiars only.

The composition of the A3921- $(\mathrm{A}+\mathrm{B}+\mathrm{C})$ sample objects identified as non-cluster members by the caustics method (see Sect. 3.1) with respect to the morphological classification is as 
F. Pranger et al.: The galaxy population of the complex cluster system Abell 3921

Table 4. Concentration index (C), Gini coefficient (G) and the second order moment of the brightest $20 \%$ of the galaxy $\left(M_{20}\right.$ index) for subclusters $\mathrm{A}$ and $\mathrm{B}$ (median values).

\begin{tabular}{lcc|c|cccc}
\hline \hline \multicolumn{7}{c}{ Subcluster A } & \multicolumn{5}{c}{ Subcluster B } \\
\hline $\begin{array}{l}R_{\mathrm{BCG} 1} \\
{[\mathrm{kpc}]}\end{array}$ & $\mathrm{C}$ & $\mathrm{G}$ & $M_{20}$ & $\begin{array}{c}R_{\mathrm{BCG} 1} \\
{[\mathrm{kpc}]}\end{array}$ & $\mathrm{C}$ & $\mathrm{G}$ & $M_{20}$ \\
\hline 934.2 & $0.437 \pm 0.015$ & $0.600 \pm 0.016$ & $-1.854 \pm 0.028$ & 942.4 & $0.427 \pm 0.008$ & $0.609 \pm 0.006$ & $-1.835 \pm 0.010$ \\
1891.5 & $0.376 \pm 0.010$ & $0.571 \pm 0.022$ & $-1.763 \pm 0.027$ & 1646.8 & $0.351 \pm 0.018$ & $0.536 \pm 0.004$ & $-1.713 \pm 0.016$ \\
2407.0 & $0.380 \pm 0.028$ & $0.553 \pm 0.028$ & $-1.813 \pm 0.055$ & 2202.4 & $0.340 \pm 0.003$ & $0.554 \pm 0.005$ & $-1.746 \pm 0.080$ \\
3388.3 & $0.370 \pm 0.011$ & $0.535 \pm 0.012$ & $-1.855 \pm 0.052$ & 3320.3 & $0.415 \pm 0.003$ & $0.556 \pm 0.007$ & $-1.861 \pm 0.005$ \\
4128.6 & $0.375 \pm 0.019$ & $0.552 \pm 0.013$ & $-1.830 \pm 0.041$ & 4375.7 & $0.397 \pm 0.010$ & $0.577 \pm 0.016$ & $-1.788 \pm 0.016$ \\
4382.5 & $0.381 \pm 0.018$ & $0.574 \pm 0.014$ & $-1.768 \pm 0.009$ & 5102.4 & $0.401 \pm 0.017$ & $0.571 \pm 0.013$ & $-1.856 \pm 0.006$ \\
\hline & & & \multicolumn{7}{c}{ Early-type galaxies } \\
\hline 156.7 & $0.571 \pm 0.009$ & $0.684 \pm 0.006$ & $-1.942 \pm 0.010$ & 814.9 & $0.592 \pm 0.006$ & $0.712 \pm 0.013$ & $-2.014 \pm 0.036$ \\
405.4 & $0.522 \pm 0.006$ & $0.649 \pm 0.006$ & $-1.978 \pm 0.016$ & 1501.7 & $0.552 \pm 0.011$ & $0.688 \pm 0.009$ & $-1.998 \pm 0.022$ \\
712.7 & $0.537 \pm 0.007$ & $0.657 \pm 0.004$ & $-2.037 \pm 0.021$ & 2212.1 & $0.546 \pm 0.019$ & $0.665 \pm 0.018$ & $-2.006 \pm 0.019$ \\
1069.2 & $0.546 \pm 0.005$ & $0.674 \pm 0.004$ & $-2.005 \pm 0.013$ & 2916.4 & $0.543 \pm 0.013$ & $0.663 \pm 0.008$ & $-2.001 \pm 0.025$ \\
2188.9 & $0.544 \pm 0.007$ & $0.659 \pm 0.004$ & $-1.992 \pm 0.025$ & 3631.6 & $0.545 \pm 0.013$ & $0.688 \pm 0.006$ & $-2.008 \pm 0.041$ \\
4279.8 & $0.548 \pm 0.010$ & $0.651 \pm 0.005$ & $-2.081 \pm 0.008$ & 5078.4 & $0.563 \pm 0.014$ & $0.676 \pm 0.013$ & $-2.028 \pm 0.015$ \\
\hline
\end{tabular}

Notes. Upper panel: late-type galaxies (subcluster A: binsize $=4$ galaxies, subcluster B: binsize $=5$ galaxies). Lower panel: early-type galaxies (binsize $=11$ galaxies). Field values (based on the whole field of view) are shown in Figs. 13-15.

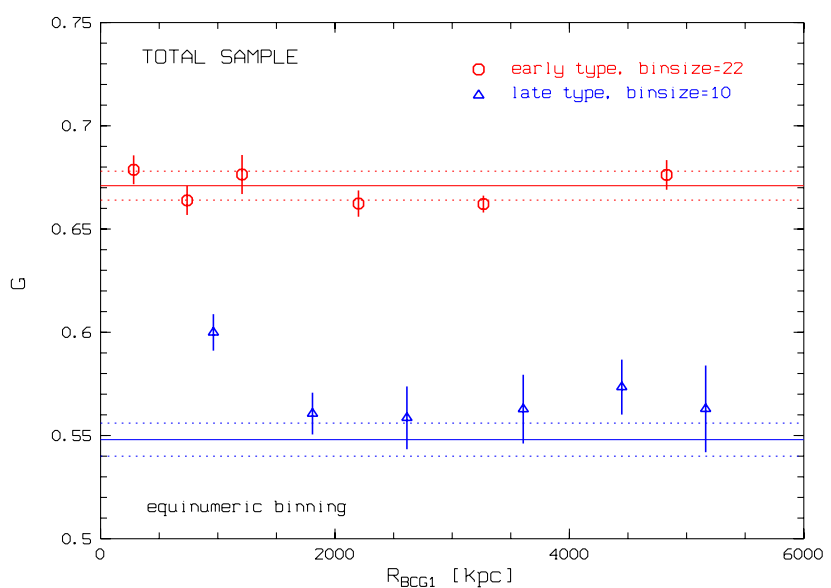

Fig. 14. Gini coefficient $G$ as a function of clustercentric distance for early-type galaxies (red circles) and late-type galaxies (blue triangles). The corresponding data for the subsamples of components $\mathrm{A}$ and $\mathrm{B}$ are listed in Table 4. The horizontal lines represent the field values and $1 \sigma$ errors.

follows: $54 \%$ ellipticals, $32 \%$ spirals, $14 \%$ peculiars (i.e. galaxies with morphological distortions), compared to a mix of $74 \%$, $25 \%$ and $1 \%$ in $A 3921-(A+B+C)$, respectively. The additional constraints implied by the caustic method would hence reduce our cluster sample by 96 galaxies showing a cluster-like distribution in $z$-space. The type mix of our sample would thus be significantly changed, in the sense that the fractions of spiral and peculiar galaxies would be reduced. Consequently, the overdensities of spirals and peculiars depicted in Figs. 19, 21 and 22 are not found when we restrict our analysis to the 119 cluster members identified using the caustics method. However, the radial trends presented in Sect. 4.2 are still found when analysing the sample of 119 cluster members, albeit with lower significance.

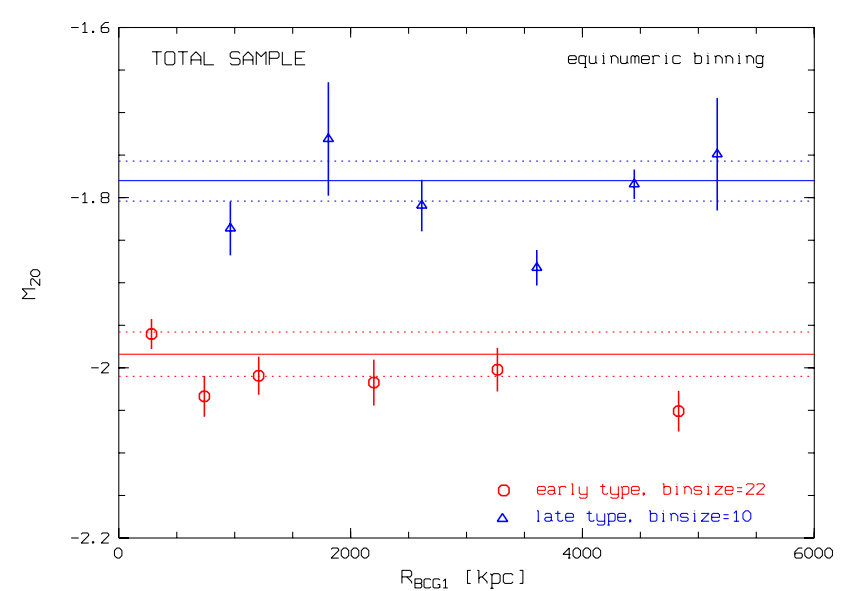

Fig. 15. Second order moment of the brightest $20 \%$ of the galaxy, $M_{20}$, as a function of clustercentric distance for early-type galaxies (red circles) and late-type galaxies (blue triangles). The corresponding data for the subsamples of components A and B are listed in Table 4. The horizontal lines represent the field values and $1 \sigma$ errors.

Note that only one of the non-cluster member objects is a spiral galaxy without detectable $[\mathrm{OII}]$ and $\mathrm{H} \alpha$ emission lines (i.e. a no-EL spiral, see Sect. 5.1).

\subsection{No-EL spirals}

On average the galaxies morphologically classified as spirals with no detectable [OII] or $\mathrm{H} \alpha$ emission are found to be closer to the cluster centre. This trend is depicted in Figs. 16 and 17. At a distance of around $3.5 \mathrm{Mpc}$ we find that the local decrease in Fig. 17 (with a corresponding increase in no-EL spiral frequency in Fig. 16) coincides with similar trends in fractions of EL-galaxies, equivalent width, SFR, and most prominent, 


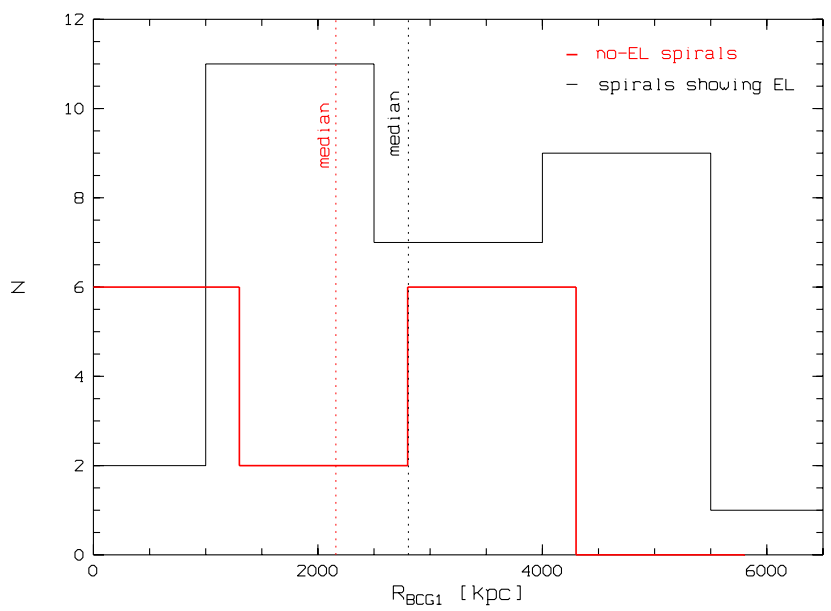

Fig. 16. Number of morphologically classified spiral galaxies as a function of clustercentric distance. The sample showing no emission lines is found to be on average closer to the cluster centre. Note that component $\mathrm{C}$ is included in this plot.

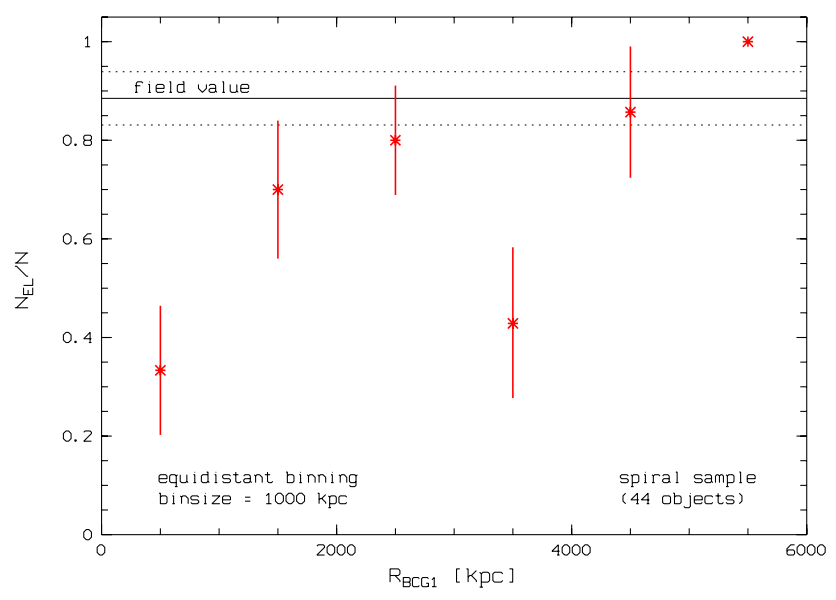

Fig. 17. Fraction of morphologically identified spirals showing [OII] or $\mathrm{H} \alpha$ emission lines, respectively. Note that component $\mathrm{C}$ is included in this plot. Data bins are equidistant.

in $M_{20}$, both in A3921-A and A3921-B. We now take a look at local projected surface number densities to determine the morphological galaxy species that come into question to be the cause for this local decrease.

\section{Surface number densities}

Figure 18 illustrates the projected absolute surface number density of the whole cluster sample including component $\mathrm{C}$ (215 galaxies, A3921- $(\mathrm{A}+\mathrm{B}+\mathrm{C}))$ based on a subdivision of the $2 \mathrm{dF}$ field-of-view into 625 equally-sized cells. From this plot we learn that the cluster seems to be elongated in south-east/northwest direction whereas some regions of high galaxy number density show an orientation from the cluster centre along a perpendicular axis, i.e. north-east/south-west. Note that this density map has been computed from the spectroscopic sample alone. Due to the limitations of maximum fibre density, it does not necessarily represent the distribution of the underlying total galaxy population.

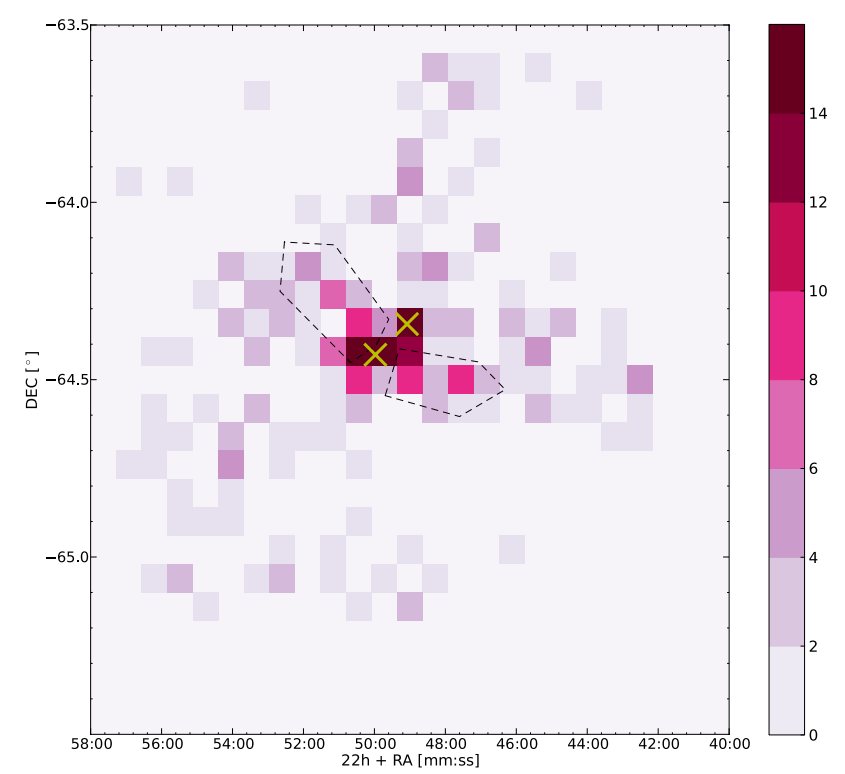

Fig. 18. Galaxy number density of the whole spectroscopic sample of A3921 (including component C). The field-of-view is subdivided into $25 \times 25$ equally-sized rectangles. The colour scale shows the number of galaxies per $\mathrm{Mpc}^{2}$. BCG1 and BCG2 are represented by yellow crosses. Overdense regions protruding from the cluster centre are enclosed by dashed lines.

\subsection{Local fractions of surface number densities}

Revisiting the unexpected trends in the radial plots (fractions of EL-galaxies, equivalent widths, SFR, $M_{20}$, fractions of no-EL spirals) at distances of $3 \mathrm{Mpc}$ to $4 \mathrm{Mpc}$ from the cluster centre we investigate the local fraction of various morphological galaxy types. Figure 19 shows the local fraction of spiral galaxies (objects from the spiral sample, see Sect. 5) relative to the extended morphological sample (EMS) within a radius of $600 \mathrm{kpc}$ around each cluster member galaxy. This circle size represents the best compromise between spatial resolution and number of galaxies per resolution element. Note that, for the sake of clarity, the symbol sizes do not correspond to a size of $600 \mathrm{kpc}$ but are much smaller. In accordance with previous studies on the abundance of galaxy species with respect to their environment (e.g. Gallazzi et al. 2008) we find a distinct deficiency of spiral galaxies in the cluster centre. Their local fraction reaches values between 0.5 and 0.6 at clustercentric distances greater than $r_{200}$. This behaviour reflects the well-known morphology-density relation found by Dressler (1980). However, we also find areas with local fractions $>0.7$ to the north-west and to the south-east of our field-of-view, both at a distance between $3 \mathrm{Mpc}$ and $4 \mathrm{Mpc}$ from the cluster centre. We do not find an increased fraction of spiral galaxies in the overdense regions indicated in Fig. 18.

The local fraction of no-EL spirals, i.e. morphologically classified spirals without detectable [OII] or $\mathrm{H} \alpha$ emission, with respect to the total spiral sample is depicted in Fig. 20. According to previous results (e.g. Bösch et al. 2013; Vogt et al. 2004) this species of galaxies is expected to be found near the cluster centre for relaxed clusters. However, A3921 shows relatively high local fractions of no-EL spirals not only in its core but also in certain areas found at about 2.9-4.2 Mpc to the northwest and to the south-east of the cluster centre.

We remind the reader that "centre" here refers to the position of BCG1 which is also justified by X-ray data on the central 30' of A3921 (Belsole et al. 2005). These data reveal just a slight shift of $17^{\prime \prime}$ between the peak in X-ray intensity and the 


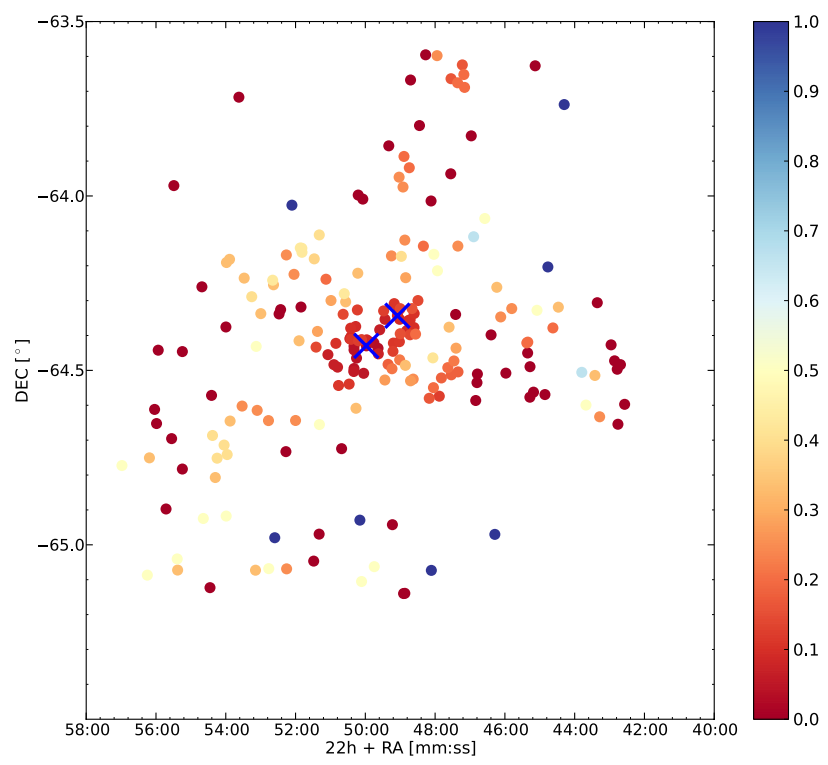

Fig. 19. Local fractions of spiral galaxies. Note the deficiency of spirals in the cluster centre and their relatively high abundance at low galaxy number densities. BCG1 and BCG2 are represented by blue crosses. Note that the values associated with the colour bar are fractions of densities and therefore have no unit.

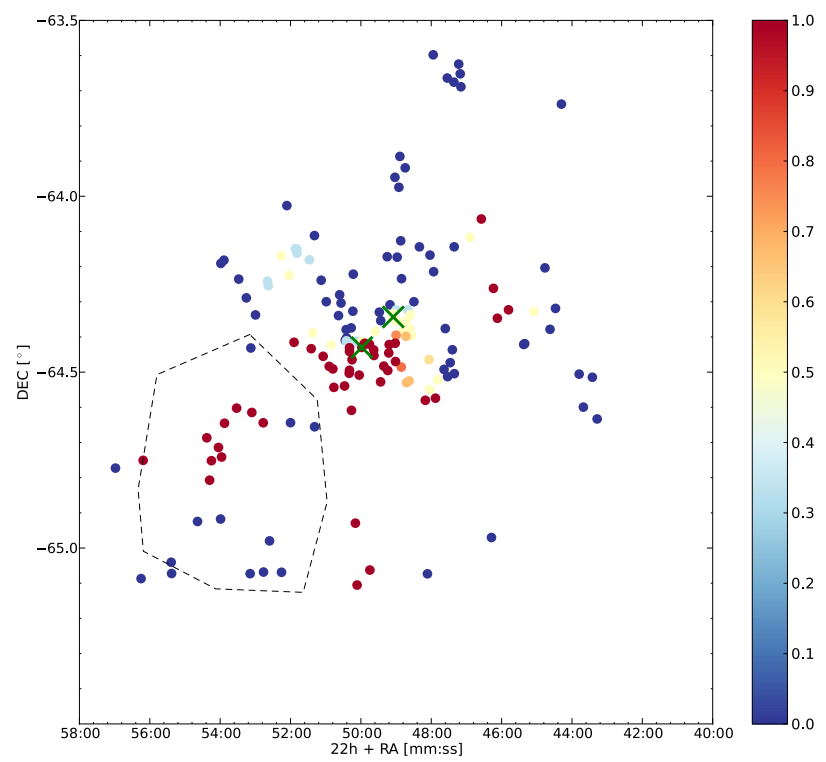

Fig. 20. Local fractions of no-EL spiral galaxies (with respect to the spiral sample). Note the relative high abundance of no-EL spirals in areas to the north-west and to the south-east of the cluster centre. BCG1 and $\mathrm{BCG} 2$ are represented by green crosses, objects assigned to component $\mathrm{C}$ are found within the area enclosed by the dashed lines. Also note that the values associated with the colour bar are fractions and therefore have no unit. The colour bar is inverted with respect to Fig. 19.

position of BCG1. X-ray residuals remaining after the subtraction of a $2 \mathrm{D} \beta$-model are found in the vicinity and to the south of BCG2 showing elongations towards BCG1 and peaking to the south-west of BCG2 - thereby giving rise to the hypothesis of an off-axis merger between A3921-A and A3921-B along southeast/north-west direction in the plane of the sky (Belsole et al. 2005).

It can be seen from Fig. 12 that the morphological classification included peculiar galaxies, i.e. galaxies showing disturbed morphology. Figure 21 shows the local fraction of

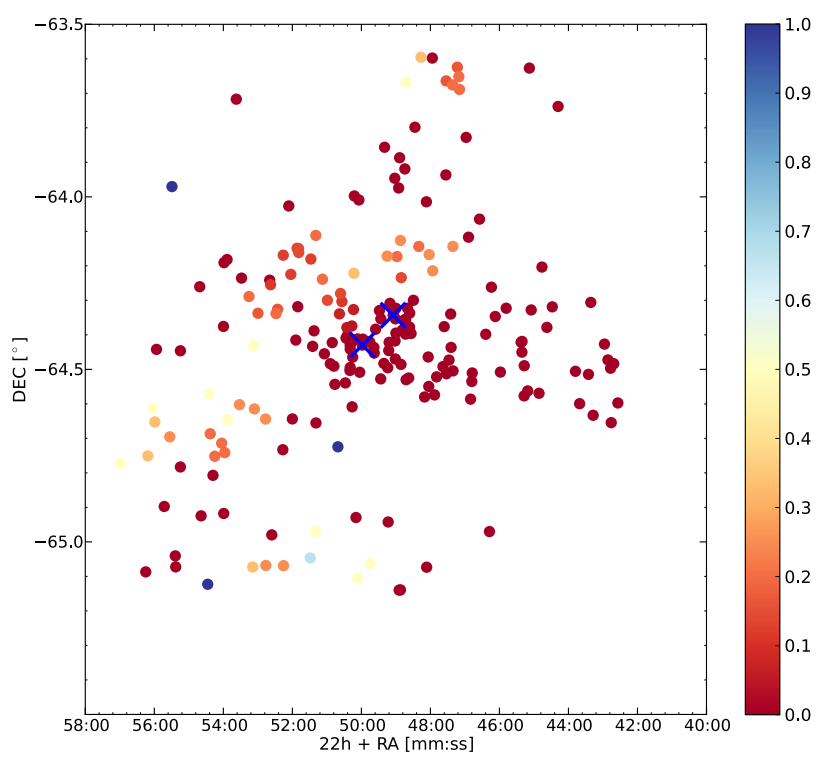

Fig. 21. Local fractions of peculiar galaxies. Note the relative high abundance of peculiars in areas to the south-east of the cluster centre as well as the deficiency of peculiars in the western half of our fieldof-view. BCG1 and BCG2 are represented by blue crosses. Also note that the values associated with the colour bar are fractions and therefore have no unit.

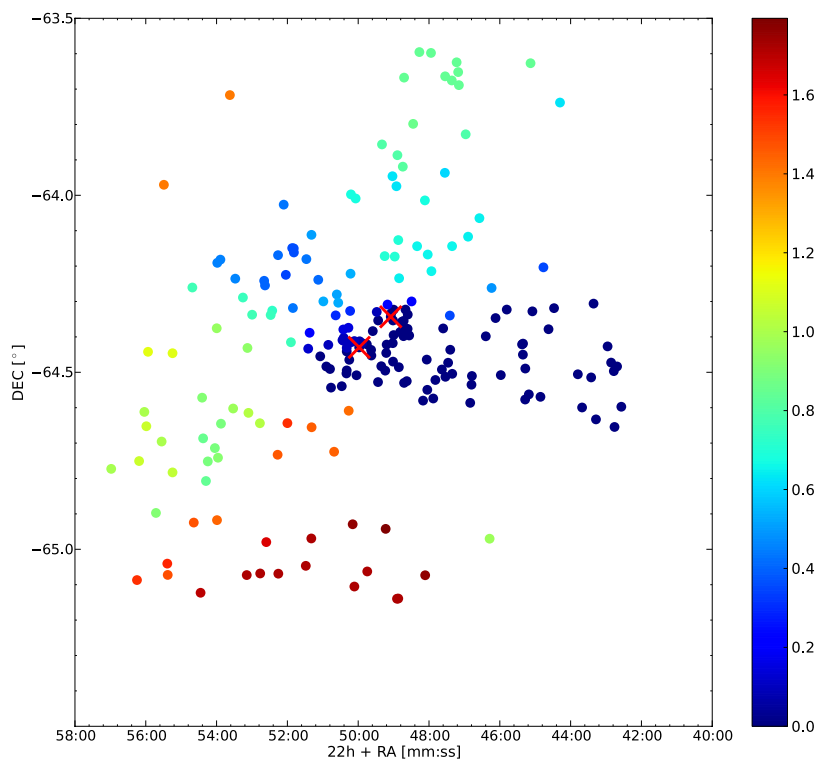

Fig. 22. Standard deviation (computed amongst 14 next neighbours, normalised to the standard deviation of the whole EMS) of the local fractions of peculiar galaxies. Note the accumulation of objects with high values in areas to the south-east of the cluster centre. BCG1 and BCG2 are represented by red crosses. The values associated with the colour bar are normalised and therefore have no unit.

peculiar galaxies relative to the extended morphological sample. Peculiars seem to be over-represented in areas to the south-east of the cluster core. In Fig. 22 the colour code represents the standard deviation (for a given galaxy and its 14 next neighbours) of the local fraction of peculiars with respect to the extended morphological sample. The values are normalised to the standard deviation of the whole distribution which is $\sim 0.175$ (with a mean value of $\sim 0.086$ ). 


\section{Discussion}

Our analyses of spectroscopical and photometric observations of 200 galaxies in the merging cluster Abell 3921 confirm the presence of two BCGs at a projected separation of $\sim 850 \mathrm{kpc}$. Each of these giant elliptical galaxies is associated with one of the merging subclusters (A3921-A, A3921-B) as pointed out in Ferrari et al. (2005). We also find the subclusters to reside at similar median redshifts of $\sim 0.093$ which is in compliance with the scenario of a merger axis almost parallel to the plane of the sky (Belsole et al. 2005; Ferrari et al. 2005). A Dressler-Shectman test reveals a third cluster component (A3921-C) in the southeast region of A3921-A. Within our sample we find 16 galaxies which are likely to be members of this component. Given its median redshift of 0.086 we argue that A3921-C is falling onto the system A3921- $(\mathrm{A}+\mathrm{B})$ from a greater radial distance. Its high fraction of peculiar galaxies yields tentative evidence that A3921-C might be a large galaxy group or even consist of several groups.

Our mass estimates for A3921-A and A3921-B exceed the values found by Ferrari et al. (2005) and Belsole et al. (2005). The detection of A3921-C lowers the mass estimate for A3921A with respect to A3921-B. Hence we find a mass ratio of A3921-B and A3921-A of 1:2 whereas Ferrari et al. (2005) find a mass ratio of $\sim 1: 5$. However, this does not contradict their favoured scenario of an off-axis merger between objects of unequal mass (with A3921-A being the more massive object) in its central phase $\left(t_{0} \sim \pm 0.3 \mathrm{Gyr}\right.$ where $t_{0}=0$ denotes the time of coalescence). In a follow-up investigation Kapferer et al. (2006) compare the ICM metallicities of A3921 (Belsole et al. 2005) to numerically simulated cluster mergers. Belsole et al. (2005) find the highest metallicity in the region of A3921-B and further to the west. Assuming that ejected metals follow behind their ejectors, Kapferer et al. (2006) hence favour a pre-merger scenario, i.e. $t_{0} \sim-0.3$ Gyr. Our new mass ratio and total mass estimates are in compliance with this scenario.

Our estimate for $r_{200}$ of A3921- $(\mathrm{A}+\mathrm{B})$ exceeds the values given by Ferrari et al. (2005) but is in excellent agreement with the results of the caustics method applied to a larger galaxy catalogue of A3921 constructed from literature data. However, our mass estimate clearly exceeds the caustic results. This discrepancy certainly is due to the fact that our mass estimates are based on the assumption of a virialised galaxy population which might not be fulfilled for A3921.

The radial trends in fractions of EL-galaxies, equivalent widths, SFRs, concentration index, Gini coefficient and $M_{20}$ up to clustercentric distances of $\sim r_{200}$ correspond to the behaviour suggested by the morphology-density relation. Fractions of EL-galaxies (and EL-spirals), equivalent widths, SFRs and $M_{20}$ surprisingly show a decrease (in absolute numbers) at $\sim 3.5 \mathrm{Mpc}$ clustercentric distance. The reason for these decreases are high fractions of no-EL spirals at radial distances of $3 \mathrm{Mpc}$ to $4 \mathrm{Mpc}$ in regions to the north-west and south-east of the cluster centre. Note that this result is robust against the definition of cluster membership: even if we restrict our analysis to virialised cluster members according to the caustics approach, the subsample of no-EL spirals is reduced by only one object (whereas the total sample of cluster members is reduced by 96 galaxies).

The highest fractions of no-EL spirals are, however, found in the cluster core at radial distances smaller than $1.2 \mathrm{Mpc}$. Analysing a volume-limited sample of SDSS data, also e.g. Goto et al. (2003) find that no-EL spirals (or "passive" spirals) mainly occur in high-density environments. Regarding the high probability of dynamical interaction processes (e.g. galaxy mergers or galaxy-galaxy tidal interactions) to disturb or destroy spiral arms Goto et al. (2003) propose a cluster related mechanism to explain their findings. According to more recent results (e.g. Bösch et al. 2013) no-EL spirals are expected to occur near the cluster centre for relaxed clusters. The generally adopted scenario is that no-EL spirals close to the cluster centre have been subject to ram pressure stripping during their infall. Quilis et al. (2000) show that on a time scale of $\sim 100$ Myr ram pressure stripping may remove the majority of a galaxy's atomic hydrogen content, thereby quenching star formation. Vogt et al. (2004) argued that no-EL spirals (or "quenched" spirals) could represent an intermediate stage of a morphological transformation of spiral galaxies (falling into the cluster from the field) into S0s. In their extensive analysis of two virialised clusters at $z \sim 0.5$ Moran et al. (2007) address the question of a transition from spiral galaxies into S0s. They find the highest fractions of passive spirals and S0s close to the cluster centres but also observe both species to reside in infalling groups out to clustercentric radii beyond $r_{200}$. The authors conclude that a combination of ram pressure stripping and galaxy-galaxy tidal interactions is required to explain their observations. The resolution of our imaging data is, however, insufficient to identify S0 galaxies. Nevertheless, the large fractions of no-EL spirals observed at small clustercentric radii in Abell 3921 are in compliance with the transformation scenario.

No-EL spirals that occur at clustercentric distances greater than $3 \mathrm{Mpc}$ are found in the directions of A3921-B to the northwest and A3921-C to the south-east. This explains why we find decreases at 3-4 Mpc in the aforementioned radial trends not only in the total cluster sample but also in subsamples A3921-A and A3921-B. The occurrence of no-EL spirals at large clustercentric radii could be explained by shocks in the ICM due to the ongoing cluster merger. A merger shock front represents a steep increase in local ICM density, pressure and temperature. A shock front moving through a galaxy (or vice versa) hence could be the site for an increased ram pressure effect. Ram pressure stripping can increase the SFR on short timescales (e.g. Steinhauser et al. 2012) and decrease it on longer timescales due to gas consumption (e.g. Quilis et al. 2000). Likewise, the influence of a shock front probably leads to a SFR decrease on long timescales. Chung et al. (2009) investigated the $6.2 \mu \mathrm{m}$ polycyclic aromatic hydrocarbon emission feature of galaxies behind and in front of a merger shock front in the "Bullet cluster". In particular, they focussed on the flux ratio of the $8 \mu \mathrm{m}$ band (containing the $6.2 \mu \mathrm{m}$ feature at the observed redshift) and the $4.5 \mu \mathrm{m}$ band which is a proxy for stellar mass. The $m_{4.5}-m_{8}$ colour was hence used as a proxy of specific SFR. Chung et al. (2009) find that postshock galaxies have on average a lower specific SFR than their pre-shock counterparts. Applying a standard KS test they find that the difference in colour between both samples is approximately of $2 \sigma$. They conclude that a shock front induced by a merger event as represented by the bullet cluster does not drastically quench star formation. However, the observed difference between galaxies in pre- and post-shock regions is significant. Evaluating data on the major merger Abell 2744, Owers et al. (2012) find very bright star forming knots and filaments in the stripped wake of four infalling spiral galaxies. Three of these so-called "jellyfish" objects are found close to merger-related features in the ICM. Hence Owers et al. (2012) suggest that the star formation in the stripped wakes has been triggered by the increase of pressure during an interaction with a merger shock. They consider their findings as evidence for the accelerated morphological transformation of galaxies due to shock interaction. As observations of A3921 in radio (ATCA, Ferrari et al. 2006) 
and X-ray (XMM-Newton, Belsole et al. 2005) did only cover regions at clustercentric distances $\lesssim 15^{\prime}$ without detecting any traces of shock fronts, our hypothesis of shocks as the reason for quenched spirals at large clustercentric radii is, to some extent, speculative. However, the existing radio and X-ray data did not cover radii out to $\sim 3-4 \mathrm{Mpc}$ where we observe the quenched spirals.

\section{Conclusions}

In summary, our spectroscopical and morphological analyses motivate the following conclusions:

- Component $\mathrm{C}$ might be a massive group falling onto the cluster from the south-east (projected onto the plane of the sky) with a high peculiar line-of-sight velocity towards the observer. The high fraction (25\%) of peculiar galaxies in component $\mathrm{C}$ would be very unusual for a cluster.

- Component $\mathrm{C}$ might be further extended in westward direction than indicated by the DS-test. This is suggested by e.g. the widespread distribution of peculiar galaxies in the respective region.

- The clustercentric distances and the locations in $z$-space of the areas north-west and south-east of the cluster centre showing overdensities of spirals, no-EL spirals and peculiar galaxies suggest that the merger between A3921-A and A3921-B is dynamically more advanced than the merger of A3921- $(\mathrm{A}+\mathrm{B})$ and component $\mathrm{C}$.

- The high fraction of no-EL spirals in the cluster core supports the hypotheses of morphological transformation in regions of increased density. According to this scenario no-EL spirals represent a transition stage between field spirals and cluster SOs.

- The unexpected occurrence of no-EL spirals at distances $>2 r_{200}$ could at least partially be explained by shock fronts in the ICM due to the ongoing cluster merger.

Acknowledgements. We thank the referee, Florence Durret, for her constructive comments which helped a lot to improve the manuscript. We warmly thank all the AAO team and in particular Rob Sharp and Fred Watson for having performed and pre-reduced our $2 \mathrm{dF}$ observations. We are very grateful to Tom Mauch for having provided the code runz. Florian Pranger, Asmus Böhm and Sabine Schindler are grateful for funding by the Austrian Funding Organisation FWF through grant P23946-N16. Chiara Ferrari acknowledges financial support by the "Agence Nationale de la Recherche" through grant ANR-09-JCJC0001-01. Antonaldo Diaferio acknowledges partial support from the INFN grant PD51.

\section{References}

Abadi, M. G., Moore, B., \& Bower, R. G. 1999, MNRAS, 308, 947 Abraham, R. G., van den Bergh, S., \& Nair, P. 2003, ApJ, 588, 218 Balogh, M. L., Navarro, J. F., \& Morris, S. L. 2000, ApJ, 540, 113 Barrena, R., Boschin, W., Girardi, M., \& Spolaor, M. 2007, A\&A, 467, 37 Beers, T. C., Flynn, K., \& Gebhardt, K. 1990, AJ, 100, 32

Belsole, E., Sauvageot, J.-L., Pratt, G. W., \& Bourdin, H. 2005, A\&A, 430, 385
Berrier, J. C., Stewart, K. R., Bullock, J. S., et al. 2009, ApJ, 690, 1292 Bertin, E., \& Arnouts, S. 1996, A\&AS, 117, 393

Bösch, B., Böhm, A., Wolf, C., et al. 2013, A\&A, 549, A142

Boselli, A., \& Gavazzi, G. 2006, PASP, 118, 517

Carlberg, R. G., Yee, H. K. C., \& Ellingson, E. 1997, ApJ, 478, 462

Chung, S. M., Gonzalez, A. H., Clowe, D., et al. 2009, ApJ, 691, 963

Colless, M., Dalton, G., Maddox, S., et al. 2001, MNRAS, 328, 1039

da Costa, L. N., Pellegrini, P. S., Davis, M., et al. 1991, ApJS, 75, 935

Dalton, G. B., Efstathiou, G., Maddox, S. J., \& Sutherland, W. J. 1994, MNRAS, 269,151

Diaferio, A. 1999, MNRAS, 309, 610

Donnelly, R. H., Forman, W., Jones, C., et al. 2001, ApJ, 562, 254

Dressler, A. 1980, ApJ, 236, 351

Dressler, A., \& Shectman, S. A. 1988, AJ, 95, 985

Ferrari, C., Benoist, C., Maurogordato, S., et al. 2004, in Outskirts of Galaxy Clusters: Intense Life in the Suburbs, ed. A. Diaferio, IAU Colloq., 195, 188

Ferrari, C., Benoist, C., Maurogordato, S., Cappi, A., \& Slezak, E. 2005, A\&A, 430,19

Ferrari, C., Hunstead, R. W., Feretti, L., Maurogordato, S., \& Schindler, S. 2006, A\&A, 457, 21

Fujita, Y. 2004, PASJ, 56, 29

Gallazzi, A., Brinchmann, J., Charlot, S., \& White, S. D. M. 2008, MNRAS, 383,1439

Geller, M. J., \& Huchra, J. P. 1989, Science, 246, 897

Goto, T., Okamura, S., Sekiguchi, M., et al. 2003, PASJ, 55, 757

Kantharia, N. G., Rao, A. P., \& Sirothia, S. K. 2008, MNRAS, 383, 173

Kapferer, W., Ferrari, C., Domainko, W., et al. 2006, A\&A, 447, 827

Kapferer, W., Sluka, C., Schindler, S., Ferrari, C., \& Ziegler, B. 2009, A\&A, 499, 87

Katgert, P., Mazure, A., Perea, J., et al. 1996, A\&A, 310, 8

Katgert, P., Mazure, A., den Hartog, R., et al. 1998, A\&AS, 129, 399

Kennicutt, Jr., R. C. 1992a, ApJS, 79, 255

Kennicutt, Jr., R. C. 1992b, ApJ, 388, 310

Lewis, I. J., Cannon, R. D., Taylor, K., et al. 2002, MNRAS, 333, 279

Lorenz, M. O. 1905, PASA, 9, Number 70, 9, 209

Lotz, J. M., Primack, J., \& Madau, P. 2004, AJ, 128, 163

Markevitch, M., Gonzalez, A. H., David, L., et al. 2002, ApJ, 567, L27

Mathewson, D. S., \& Ford, V. L. 1996, ApJS, 107, 97

McGee, S. L., Balogh, M. L., Bower, R. G., Font, A. S., \& McCarthy, I. G. 2009, MNRAS, 400, 937

Mendes de Oliveira, C., \& Hickson, P. 1994, ApJ, 427, 684

Moore, B., Katz, N., Lake, G., Dressler, A., \& Oemler, A. 1996, Nature, 379, 613

Moran, S. M., Ellis, R. S., Treu, T., et al. 2007, ApJ, 671, 1503

Muriel, H., Quintana, H., Infante, L., Lambas, D. G., \& Way, M. J. 2002, AJ, 124,1934

Owers, M. S., Couch, W. J., Nulsen, P. E. J., \& Randall, S. W. 2012, ApJ, 750, L23

Paul, S., Iapichino, L., Miniati, F., Bagchi, J., \& Mannheim, K. 2011, ApJ, 726, 17

Pimbblet, K. A., Smail, I., Edge, A. C., et al. 2006, MNRAS, 366, 645

Porter, S. C., \& Raychaudhury, S. 2007, MNRAS, 375, 1409

Quilis, V., Moore, B., \& Bower, R. 2000, Science, 288, 1617

Roettiger, K., Burns, J. O., \& Loken, C. 1996, ApJ, 473, 651

Serra, A. L., \& Diaferio, A. 2013, ApJ, 768, 116

Serra, A. L., Diaferio, A., Murante, G., \& Borgani, S. 2011, MNRAS, 412, 800

Small, T. A., Ma, C.-P., Sargent, W. L. W., \& Hamilton, D. 1998, ApJ, 492, 45

Steinhauser, D., Haider, M., Kapferer, W., \& Schindler, S. 2012, A\&A, 544, A54

Tonry, J., \& Davis, M. 1979, AJ, 84, 1511

Toomre, A., \& Toomre, J. 1972, ApJ, 178, 623

Verdugo, M., Ziegler, B. L., \& Gerken, B. 2008, A\&A, 486, 9

Vogt, N. P., Haynes, M. P., Giovanelli, R., \& Herter, T. 2004, AJ, 127, 3300

Way, M. J., Quintana, H., Infante, L., Lambas, D. G., \& Muriel, H. 2005, AJ, 130,2012

Yahil, A., \& Vidal, N. V. 1977, ApJ, 214, 347

Zabludoff, A. I., Geller, M. J., Huchra, J. P., \& Vogeley, M. S. 1993, AJ, 106, 1273 